\title{
Initial Synchronisation of Wideband and UWB Direct Sequence Systems: Single- and Multiple-Antenna Aided Solutions
}

\author{
SeungHwan Won and Lajos Hanzo
}

\begin{abstract}
This survey guides the reader through the open literature on the principle of initial synchronisation in singleantenna-assisted single- and multi-carrier Code Division Multiple Access (CDMA) as well as Direct Sequence-Ultra WideBand (DS-UWB) systems, with special emphasis on the DownLink (DL). There is a paucity of up-to-date surveys and review articles on initial synchronization solutions for MIMO-aided and cooperative systems - even though there is a plethora of papers on both MIMOs and on cooperative systems, which assume perfect synchronization. Hence this paper aims to fill the related gap in the literature.
\end{abstract}

Index Terms-CDMA, Code acquisition, Initial synchronisation, MIMO, UWB.

\section{INTRODUCTION}

$\mathbf{C}$ ODE Division Multiple Access (CDMA) [1] based mobile communication systems have been widely advocated by the standardisation bodies, leading to the IS-95 [1] and the Third-Generation (3G) [2] systems. In the inter-cell synchronous CDMA-2000 system, the Mobile Station's (MS) receiver must be capable of coarsely synchronously aligning a locally generated Pseudo-Noise (PN) code with the received composite multi-user signals containing the desired user's PN sequence [3]-[6] before the transmission of the desired signal. This process is referred to as 'initial code acquisition', followed by optimum post-initial code acquisition [7], [8], where the term 'post-initial code acquisition' [7] refers to identifying the timing instants of the affordable-complexitydependent number of delayed received signal paths, which will be combined by Rake receiver.

In general, the initial synchronisation is achieved in two consecutive steps. Code acquisition is invoked first for coarse code alignment and code phase tracking for fine alignment [3], [5], [9]. Substantial research efforts have been devoted to the design of code acquisition techniques [4]-[6]. A variety of serial search [3], [4], parallel search [10], [11], as well as sequential estimation [12]-[14] based code acquisition techniques have been proposed in the literature. In order to achieve a high performance, numerous detector structures such as multiple dwell based search schemes [3]-[5], PostDetection Integration (PDI) [3], [15], [16] and Differentially

Manuscript received 1 March 2010; revised 12 September 2010. The financial support of both the RC-U.K. under the auspices of the India-UK Advanced Technology Centre and that of the EU under the auspices of the Optimix project is gratefully acknowledged.

The authors are with the School of ECS, University of Southampton, SO17 1BJ, United Kingdom (e-mail: lh@ecs.soton.ac.uk).

Digital Object Identifier 10.1109/SURV.2011.101310.00028
Coherent (DC) [17]-[19] schemes have also been studied in the literature. Then, the code acquisition performance of Multi-Carrier (MC) DS-CDMA evaluated with the aid of serial and parallel search based schemes has been investigated in [6], [20], [21], respectively.

Most code acquisition schemes have originally been designed for Single-Input Single-Output (SISO) systems [3], [16], [22]. However, there is a paucity of in-depth studies in the open literature representing the fundamental characteristics of code acquisition schemes, regardless whether co-located or cooperative Multiple Input/Multiple Output (MIMO) scenarios considered. Hence, we have reviewed a variety of code acquisition schemes designed for antenna arrays, since they may be considered an important generic MIMO family [23] [27]. The recent contributions disseminated in [28]-[33] aimed for the fundamental characterization of code acquisition schemes assisted by either co-located or cooperative MIMOs. Furthermore, the research of Ultra WideBand (UWB) systems has recently attracted a significant interest in both the academic and industrial research community [34], [35]. However, initial code and timing acquisition in the DS-UWB DL constitutes a particularly challenging problem owing to the extremely short signalling chip-duration employed [36], [37]. Accordingly, in this survey we dedicate special attention to the employment of iterative code acquisition schemes designed for diverse MIMOs.

In Section II we commence our discourse by introducing the related terminology, definitions and various approaches regarding code acquisition. In Section III we guide the reader through the extensive literature, commencing from the conception of the various schemes and portray a variety of search strategies as well as schemes, leading to the concept of initial and post-initial code acquisition concepts appliable to both single-carrier and MC-DS-CDMA systems. In Section IV a brief historical perspective is provided, leading to the current state-of-the-art associated with code acquisition schemes employed in DS-UWB systems. We then proceed further to provide insights on the pertinent issues associated with the family of iterative Message Passing (MP) aided acquisition approaches. The associated performance versus complexity trade-offs are illustrated in terms of serial, hybrid, parallel and iterative MP acquisition schemes. Subsequently, in Section V we consider antenna array aided MIMO scenarios as well as both co-located and cooperative MIMO environments. Then, in Section VI we detail a range of current research endeavors 
TABLE I

OVERALL STRUCTURE OF THE SURVEY

\begin{tabular}{|l|l|}
\hline II. Preliminaries & $\begin{array}{l}\text { A. Downlink and uplink procedures } \\
\text { B. Procedures of the synchronisation module } \\
\text { C. Decision strategy } \\
\text { D. Interpretation of the mean acquisition time }\end{array}$ \\
\hline III. Schemes & $\begin{array}{l}\text { A. Search strategy } \\
\text { B. Detector structure } \\
\text { C. Initial and post-initial acquisition } \\
\text { D. MC-DS-CDMA }\end{array}$ \\
\hline IV. DS-UWB & $\begin{array}{l}\text { A. Overview } \\
\text { B. Iterative message passing aided scheme } \\
\text { C. Two-stage iterative acquisition } \\
\text { D. Performance versus complexity }\end{array}$ \\
\hline V. Diverse MIMOs & $\begin{array}{l}\text { A. Antenna array } \\
\text { B. Co-located MIMOs }\end{array}$ \\
& $\begin{array}{l}\text { C. Cooperative MIMOs } \\
\text { D. Their chracteristics }\end{array}$ \\
\hline
\end{tabular}

and future research ideas. Finally, a glossary is provided in Section VII. The following table provides the overall structure of the survey.

\section{Preliminaries}

In this section, we will strive to elucidate, code acquisition procedures, the interaction between the synchronisation module and Rake receiver, the associated decision strategy and the concept of Mean Acquisition Time (MAT) in a simple and concise manner. Our discourse will be limited to the following topics:

- Code acquisition procedures in the DownLink (DL) and UpLink (UL);

- The procedures of the synchronisation module and Rake receiver;

- The basic principle of the decision strategy;

- Characterization of the MAT.

Each point will be treated separately in the forthcoming subsections. We would like to direct the attention of those readers, who wish to delve into further detail, to some excellent textbooks such as [3]-[6], amongst others.

\section{A. Code Acquisition Procedures in the Downlink and Uplink}

The initial processing stage of CDMA systems is the acquisition of the correct timing of the incoming signals received both in the DL and UL. The code acquisition in the DL is the first operation that has to be carried out, as soon as the MS is switched on. The operation is carried out by processing an unmodulated pilot signal, which is spread according to the the chip rate and is broadcast over the entire cell. There is no power control during this stage. In the DL, the uncertainty region (or search window width) corresponds to the entire duration of the PN sequence, which tends to be quite wide, for example $\left(2^{15}-1\right)$ chip intervals in the DL of the inter-cell synchronous CDMA-2000 system [3], [16], [38]

1 . Hence the MAT is minimised in the context of serial search

\footnotetext{
${ }^{1}$ After the Base Station (BS) transmitted the pilot to the MS, the MS responds with a preamble and hence the BS becomes aware of the system's turn-around delay, which determines the MS's distance from the BS. This technique allows the MS to advance its transmission instant by the estimated amount of the propagation delay, which in turn allows the BS to shorten its search window duration. This also allows the BS to detect immediately after the elapse of code phase verification, if a false alarm locking event was encountered.
}

techniques by achieving the best possible correct detection probability, while maintaining as low a value of the false alarm probability and false locking penalty as possible. The effect of the above-mentioned high uncertainty region of the DL could be potentially mitigated by testing the correct spreading code alignment hypotheses using parallel search techniques. ${ }^{2}$ However, given the wide uncertainty region of the DL, the implementation of the parallel search based hardware often becomes impractical due to its high complexity. ${ }^{3}$

On the other hand, in the UL code acquisition scenario, the MS transmits a short preamble in response to the BS's pilot signal. Again, this preamble is short in order to avoid consuming a high amount of energy. The MS's preamble is then aligned in time - using classic correlation techniques with the pilot signal transmitted by the BS after the previously mentioned code acquisition in the DL. The MS is capable of invoking an open-loop power control procedure by estimating the received pilot signal power in the DL and then adjusting its transmitted power. In this case no acknowledgment is received from the BS and hence, repeated transmission attempts are made by the MS using an increased transmitted power at random time instants [38]. Based upon the above-mentioned procedure, the BS instructs the MS to advance its transmission instant in order to precompensate the effect of the propagation delay imposed on the spreading code phase. The duration of the uncertainty region within which the code phase of the received preamble has to be located depends on the maximum distance between the BS and MS. Accordingly, the uncertainty region in the UL corresponds to a fraction of the entire duration of the $\left(2^{15}-1\right)$-chip PN sequence. For example, it is less than 200 PN chip intervals in the UL of the inter-cell synchronous CDMA-2000 system [40]. The BS has to recognise the correct timing of the MS's transmitted signal within the given search window duration and collect all the multi-path components available in order to achieve the highest possible Rake combining gain. Finally, it is worth emphasising that in the context of the UL the MAT is more critically dominated by achieving the best possible correct detection probability than by attaining the lowest possible false locking probability, since the detrimental effect of a false locking event is deweighted by the short duration of the UL uncertainty region [40]. Hence the correct detection probability is typically increased with the aid of a sufficiently low detection threshold at the cost of an increased false alarm probability.

\footnotetext{
${ }^{2}$ In [40] the classic parallel acquisition arrangement was referred to as the Maximum Likelihood (ML) technique.

${ }^{3}$ Even though our topics focus on code acquisition procedures used in inter-cell synchronous systems, cell search procedures designed inter-cell asynchronous systems also play a pivotal role. A brief summury of the cell search is as follows. A target cell can only be identified by assigning a unique cell-specific scrambling code. Wideband CDMA exploiting 512 DL primary scrambling codes is capable of supporting unambiguous cell identification. The process of finding a cell and synchronising to its DL scrambling code is referred to as cell search. A simple three-step cell search procedure may be formulated as: 1) slot synchronisation, 2) frame synchronisation and code group identification, and finally 3) scrambling code identification. Further reading on the topic may be found in [39].
} 


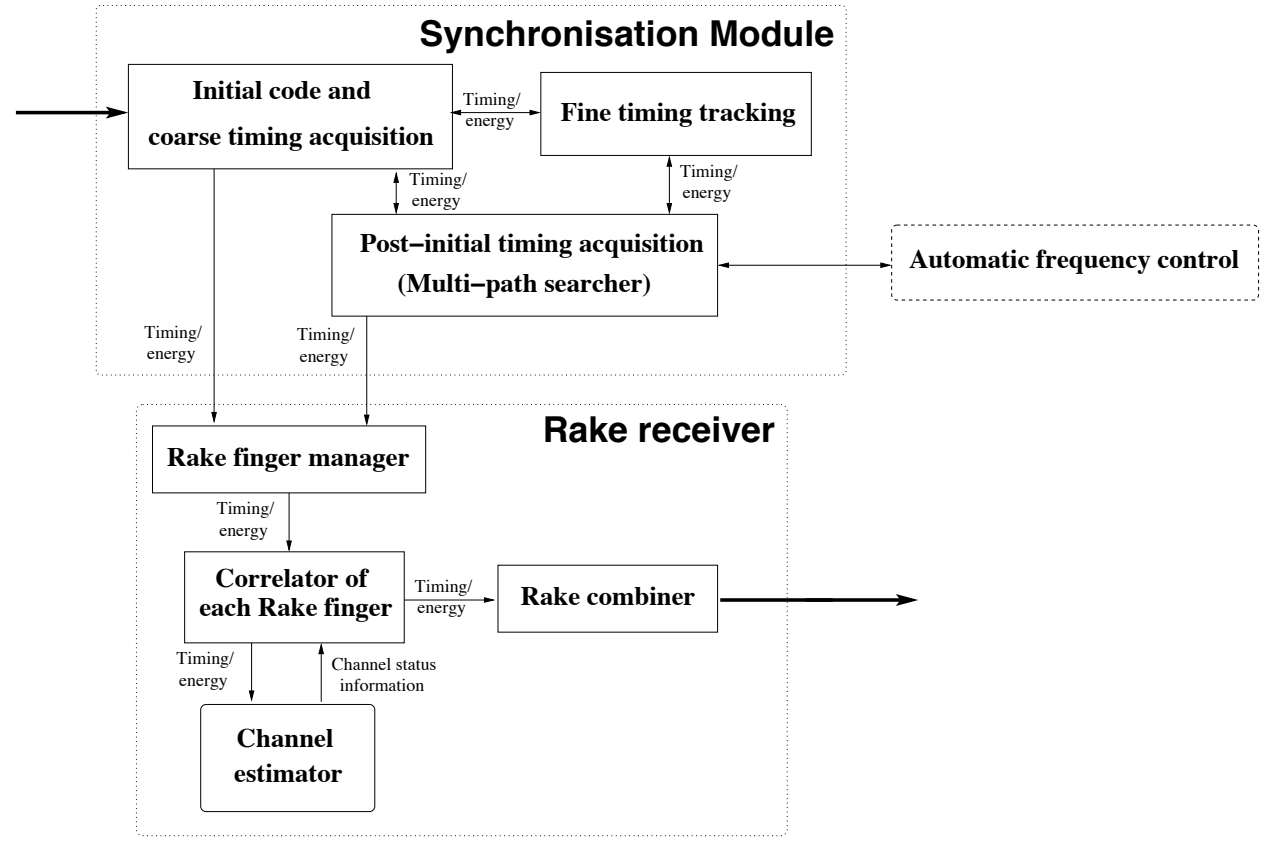

Fig. 1. Generic structure of the synchronisation module and Rake receiver of the mobile station.

\section{B. Procedures of the Synchronisation Module and Rake Receiver}

A generic structure of the synchronisation module and of the Rake receiver at the MS is highlighted in Figure 1. Explicitly, the synchronisation module at the top of Figure 1 is constituted by the combined operations of initial code acquisition, fine timing tracking and post-initial code acquisition, which is also associated with the Automatic Frequency Control (AFC). On the other hand, the Rake receiver module at the bottom of Figure 1 incorporates the Rake finger manager, each Rake finger's correlator, the Rake combiner and the channel estimator. In the inter-cell synchronous CDMA-2000 system, the MS's receiver must be capable of coarsely aligning a locally generated PN code with the received composite multiuser signals containing the desired user's PN sequence [3]-[6] before the transmission of the desired signal. After code acquisition, which ensures the above-mentioned coarse code alignment, timing tracking invoked for fine code alignment commences [3]-[5]. Following the successful initial code acquisition the AFC operation has to be activated for the sake of mitigating the total frequency mismatch, which represents the sum of the clock-drift-induced frequency mismatch between the BS's transmitter as well as the MS's receiver, and of the effect of the Doppler shift. Finally, the post-initial code acquisition procedure follows [7], in order to identify the timing instants of the affordable-complexity-dependent number of delayed received signal paths, which will be combined by the Rake receiver. The 'Rake finger manager' is capable of controlling the allocation and deallocation of Rake fingers based on both the timing and energy of the resolvable paths provided by the post-initial code acquisition stage. Based on the decisions of the Rake finger manager, all the Rake receiver's resolvable paths are coherently combined in order to achieve the best possible performance. The operational procedure of the DS-UWB system is also similar to that of the inter-cell synchronous CDMA-2000 system, except for the AFC operation, because the transmitted signal of the DSUWB is constituted by an impulse train, which is not affected by the frequency mismatch.

\section{Basic Principle of the Decision Strategy}

There are a number of ways to describe the best decision strategy in terms of the correct detection and false alarm probabilities [41]. One of the most useful strategies is constituted by the Neyman-Pearson criterion [41], which is likely to be the most important criterion in both radar and sonar detection problems. Hypothesis testing is often based on the Neyman-Pearson criterion, which seeks to minimise the probability of choosing ' 0 ' when ' 1 ' is true (a 'miss'), while maintaining an acceptable probability of choosing a ' 1 ' when a ' 0 ' is true (a 'false alarm') [3], [41]. More explicitly, this strategy chooses the decision rule by ensuring that the false alarm probability $P_{F}$ is no higher than some specified upper bound and within this constraint, maximises the probability of the correct detection $P_{D}$. Furthermore, an important benefit of this criterion is that it yields a Constant False-Alarm Rate (CFAR) [38], [41].

According to references [3], [38], [42], code acquisition can be considered to be a classical binary hypothesis testing problem, namely that of testing, whether the desired signal is deemed to be present (an $H_{1}$ hypothesis), whilst eliminating all incorrect hypotheses denoted by $H_{0}$ and at the same time trying to avoid any missed detection and false alarm events. A correlator output threshold-based decision criterion has been chosen. The correlator's decision threshold value has been optimised in the literature based upon a whole host of criteria [3], [41]. The above-mentioned NeymanPearson criterion [3], [41] has often found favour in mobile environments [3], [38], [42], since it constitutes a CFAR based approach. According to the CFAR technique [38], the 
decision variables are appropriately normalised by the estimate of the background noise plus interference variance. Hence, in case of using a CFAR technique, the various probabilities associated with the code acquisition process, including those associated with $H_{0}$ hypothesis testing do not depend on the distribution of the fading. The adoption of the CFAR based approach is particularly desirable in the specific family of code acquisition arrangements, which have to be robust against fading. In fact, it was shown in [3], [38], [42] that in hypothesis testing based upon the CFAR technique, the effects of fading channels on the code acquisition process are restricted to the testing related to the $H_{1}$ hypothesis. Furthermore, in the presence of fading, it is important to adopt the CFAR based approach for the sake of decision threshold optimisation, in order to achieve the best possible acquisition performance. As a benefit of the above-mentioned normalisation associated with the CFAR-based approach [38], the fading channel does not affect the testing of the $H_{0}$ hypotheses. The resultant scenario and the related test becomes reminiscent of an AWGN scenario. As a consequence, the fading envelope only affects the correct detection probability. The exploitation of the statistical independence of the decision variables, which is a consequence of using sufficient statistics under the assumption of employing a CFAR technique allow us to generate the analytical expressions of both the correct detection and false-alarm probabilities.

\section{Basic Principle of the Mean Acquisition Time}

In [3], [15], [22], explicit MAT formulae were provided for a single-antenna aided serial search based code acquisition system. There is no procedural difference between a singleantenna aided scheme and a multiple-antenna assisted one in terms of analysing the associated MAT, except for deriving the correct detection and the false alarm probability based upon MIMO rather than a single transmit/single receive antenna [28]-[33]. We will commence our discourse by illustrating the rudimentary concept of Single Dwell Serial Search (SDSS). The classic SDSS technique has been employed in scenarios, where the uncertainty region representing the search window width is quite wide. Hence in the context of SDSS the MAT is the vital performance criterion, as seen for example in the DL of the inter-cell synchronous CDMA-2000 system of [3], [16]. In this scheme, in the absence of any prior information such as a predetermined SDSS starting point, all the potential candidate code phases are serially searched, until the correct phase is determined. Determination of the correct code implies registering a sufficiently high correlator energy output exceeding a threshold value optimised in terms of the best possible MAT performance at the receiver. The associated trade-off is that a low threshold value may offer result in a high probability of exceeding the threshold owing to channel impairments or interference, which results in a so-called false alarm. In this case it takes a long time for the receiver to return to its search mode, hence substantially degrading the MAT. By contrast, an excessive threshold value results in a high miss probability, when the channel-contaminated reduced signal does not pass the correlation test. If the estimated code phase is deemed correct, despreading will be carried out.

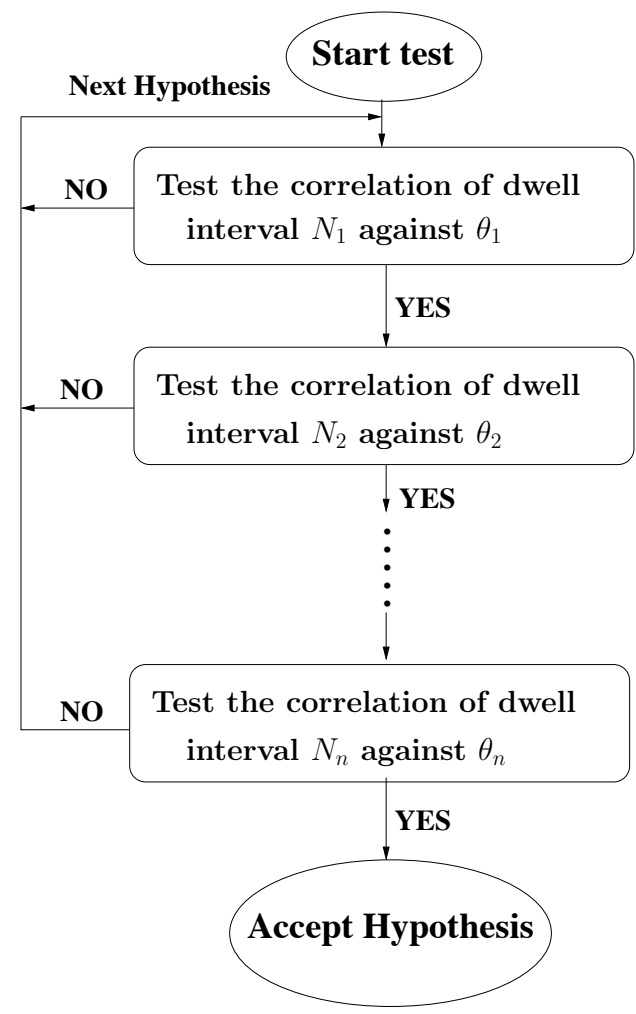

Fig. 2. Schematic diagram of multiple dwell based search system.

In SDSS [3] the receiver examines all the possible chipdelayed code phases in either a serial or parallel fashion, until the correct code phase is identified. In this scenario, the achievable MAT is typically quite high due to the full hypothesis-testing of all possible code phase. Accordingly, the achievable MAT performance of both serial search based and parallel search based code acquisition may be substantially improved by performing tests assuming multiple dwell times [3]-[5]. Since most of the so-called cells associated with specific chips correspond to incorrect code-phase alignments, their rapid elimination leads to a significant reduction of the MAT. The multiple-dwell based search may be viewed as a generalisation of the single-dwell based scheme. Figure 2 illustrates the flowchart of the multiple-dwell based search scheme, where the detector's dwell-intervals are ordered for the sake of improving the correct detection probability on the basis of how high the correlation $R\left(N_{i}\right)$ recorded for dwellinterval $N_{i}$ was, yielding $R\left(N_{1}\right) \leq R\left(N_{2}\right) \leq \ldots R\left(N_{n}\right)$. The predetermined correlation threshold values $\theta_{1} \ldots \theta_{n}$ constitute important system parameters, which have to be increased for the sake of decreasing the associated false alarm probability. It is worth noting that in practice the optimum MAT value is typically achieved in the false alarm probability range spanning from $10^{-3}$ to $10^{-4}$ in conjunction with the best possible correct detection probability, given a specific length of the coherent summation. In practice, Double Dwell Serial Search (DDSS) schemes are widely used [3], [16], since they constitute an attractive tradeoff between the achievable MAT performance and the affordable hardware complexity.

Figure 3 portrays the schematic of DDSS, where the blockdiagram shows both the search mode and the verification 


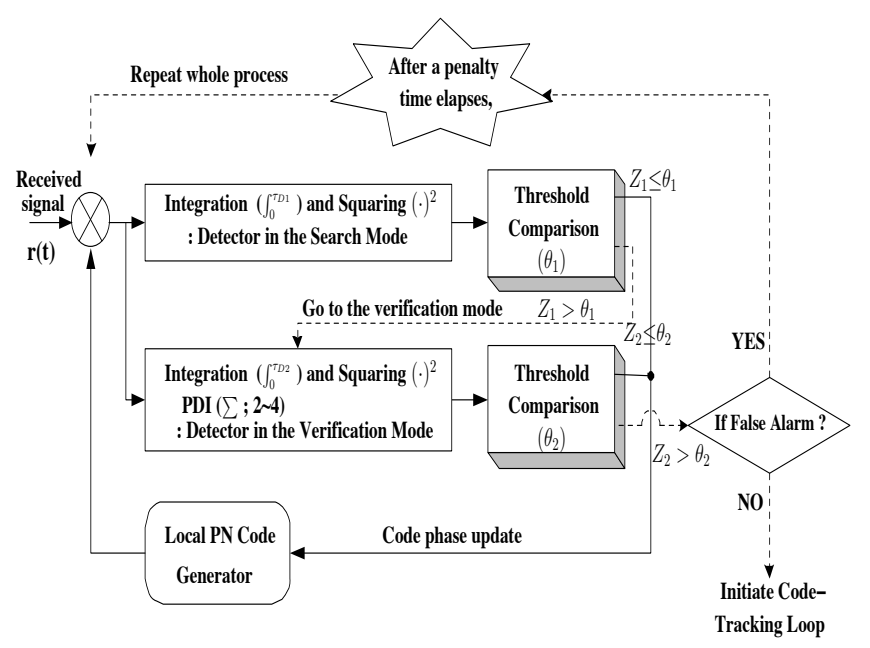

Fig. 3. Schematic diagram of double dwell serial search.

mode. More explicitly, if the correlation-test of $Z_{1}>\theta_{1}$ is satisfied, the result of the search mode in the upper branch is confirmed in the verification mode of the lower branch using the second integer dwell time $\tau_{D 2}$ and the correlator threshold of $Z_{2}>Z_{1}$. The first integration dwell time $\left(\tau_{D 1}\right)$ is defined as the time required for testing each hypothesis in the search mode which is typically on the order of 64 chips. If the estimated code phase is deemed correct in the search mode, the verification mode is activated in order to confirm, whether $H_{D}$ the previous decision is correct or not. For the sake of achieving a high-reliability decision, both the coherent integration interval and the number of PDI stages should be optimised ${ }^{4}$. If the decision is confirmed in the verification mode, the corresponding code phase can be deemed the correct code phase of the received signal. Otherwise, the search mode will be reactivated, until the correct code phase will be obtained and confirmed. In a scenario, where false alarm was encountered, after a penalty time, the search mode will be reactivated.

\section{Code Acquisition Schemes}

Following this rudimentary introduction to the associated background, we now proceed with a glimpse of the various schemes related to code acquisition. We commence this section with a discussion of various search strategies. The underlying serial search based code acquisition is presented in Subsection III.A.1, followed by the parallel search (ML) based code acquisition scheme in subsection III.A.2. Then the set of known sequential estimation based code acquisition schemes are classified into four categories in Subsection III.A.3, which includes Rapid Acquisition using Sequential Estimation (RASE), recursion-aided RASE, majority logic decoding based RASE and Recursive Soft Sequential Estimation (RSSE). Finally, in Subsection III.A.4, the random search based code acquisition is presented. Then, we introduce a

\footnotetext{
${ }^{4}$ Selection of the optimised value of the coherent integration interval is highly dependent upon the performance degradation of the carrier frequency mismatch between the BS's transmitter and the MS's receiver and a specific number of PDI stages must be determined by satisfying a pair of targeted correct detection and false alarm probabilities in terms of minimised MAT.
}

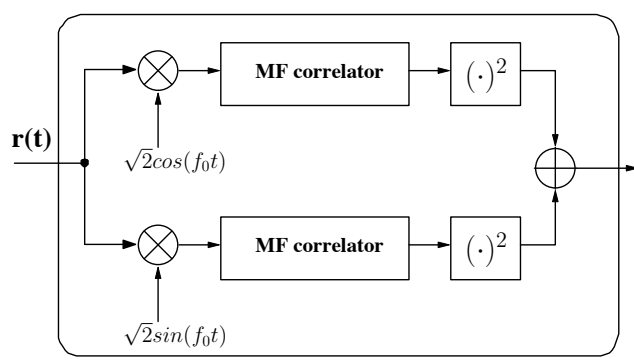

Fig. 4. Schematic diagram of the serial search based receiver.

variety of widely used detector structures in Subsection III.B. More specifically, Non-Coherent (NC) code acquisition is highlighted in Subsection III.B.1. Specifically, both a chip based-DC detection scheme and a Full-Period Correlation (FPC) based DC detection scheme are highlighted in Subsection III.B.2. In Subsection III.B.3, the widely-used PDI concept of practical code acquisition receivers is highlighted. Then, a concise portrayal of both initial and post-initial code acquisition is provided in Subsection III.C. Finally, code acquisition schemes designed for the MC-DS-CDMA DL are briefly presented in Subsection III.D. We also summarise the historic evolution of sequential estimation based code acquisition as well as that of DC acquisition schemes in Tables II and III, respectively.

\section{A. Search Strategy}

1) Serial Search Based Code Acquisition: One of the widely used initial acquisition techniques is constituted by the serial search based scheme [3]-[5]. The classic serial search technique has been employed in scenarios, where the uncertainty region (also referred to as the search window width) is quite wide and hence in the context of serial search the MAT is the vital performance criterion, as argued for the DL of the inter-cell synchronous CDMA-2000 system in [3], [16]. In this scheme, all the potential initial code phase-related time-offsets are serially searched until the correct phase is found. The correct code phase is deemed to be found, when a sufficiently high energy correlator output exceeding a threshold value optimised in terms of the achievable MAT performance is found. If the estimated code phase is deemed correct, direct-sequence despreading will be carried out. Figure 4 depicts the schematic diagram of the NC Matched-Filter (MF) based receiver designed for serial search based code acquisition. When considering more realistic multi-path fading environments, the optimal search strategies conceived for dense multi-path channels have been derived on the basis of sound mathematical foundations. A form of non-consecutive serial search capable of achieving a near-optimal mean acquisition time was proposed in [43].

2) Parallel Search Based Maximum-Likelihood Code Acquisition: One of the natural acquisition scheme extensions invoked for the sake of accomplishing an improved correct phase acquisition is to utilise a bank of $\Psi$ parallel NC Inphase (I)- Quadrature phase (Q) MFs [6], [10], [11], which are capable of searching $\Psi$ code phase positions simultaneously. As a result of employing the $\Psi$ parallel branches, the achievable MAT may be expected to decrease by a factor 


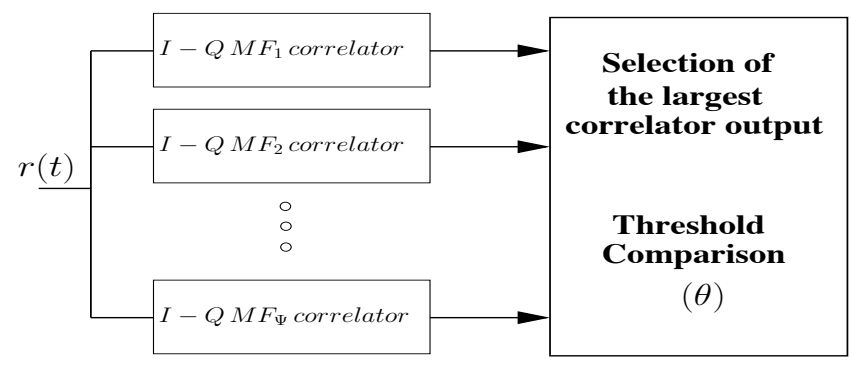

Fig. 5. Schematic diagram of the parallel search based receiver.

of $\Psi$ at the cost of a higher hardware complexity. Figure 5 portrays the schematic diagram of the resultant parallel search based receiver employing $\Psi$ parallel NC I-Q MFs [10]. Each of the $\Psi$ parallel NC I-Q MFs obeys the structure of Figure 4 [10].

The $\Xi$-chip duration of an $m$-sequence is divided into $\Psi$ shorter sequences, each having a length of $\Phi=\Xi / \Psi$ chips and then each NC I-Q MF branch is configured to acquire one of the $\Psi$ shorter sequences. The best code-phase is found for each of the $\Xi$ number of $\Phi$-chip shorter sequences and the best code-phase of the original $\Xi$-chip sequence is decided on the basis of the most reliable, i.e. highest-output $\Phi$-chip correlator of Figure 5. To elaborate a little further, the code phase associated with the highest energy correlator output among all the $\Psi$ outputs is compared to an optimised threshold value. Then, if the largest correlator output exceeds the optimised threshold value, the acquisition system declares that the codephase of the corresponding $\Phi$-chip reduced-length sequence is indeed the correct code phase of the original $\Xi$-chip sequence. If the threshold value is not exceeded, the above-mentioned process will be repeated, until a reliable $\Phi$-chip code-phase is found. It is worth noting that the shorter the reduced-length $\Phi$-chip sequences are, the higher the number of parallel branches becomes. This potentially increases the attainable acquisition speed, but reduces the reliability of detecting the correct phase of the original full-length $\Xi$-chip sequence. Accordingly, there is a trade-off between the increased speed and decreased reliability of code acquisition.

3) Sequential Estimation Based Code Acquisition: The philosophy of sequential code-phase estimation based code acquisition is similar to that of the RASE scheme of [12] which is quite attractive in terms of its achievable performance right across Signal-to-Interference plus Noise Ratio (SINR) range. Moreover, as opposed to the classic serial [3], [4] or parallel search [10], [11] based schemes, this scheme is quite vulnerable to the effect of AWGN and to interferening signals, since sequential code-phase estimation does not have an innate interference rejection capability, This is because the phase acquisition process is accomplished on the basis of a chipby chip correlation operation, rather than by exploiting the inherent structure of PN codes. By contrast, both RecursionAided RASE (RARASE) [13] and Majority Logic Decoding (MLD) based RASE [44] constitute well known enhanced versions of the original RASE scheme [12], although they still exhibit certain performance limitations. For the sake of overcoming these limitations, RSSE based acquisition schemes exploiting the inherent properties of both classic $m$-sequences

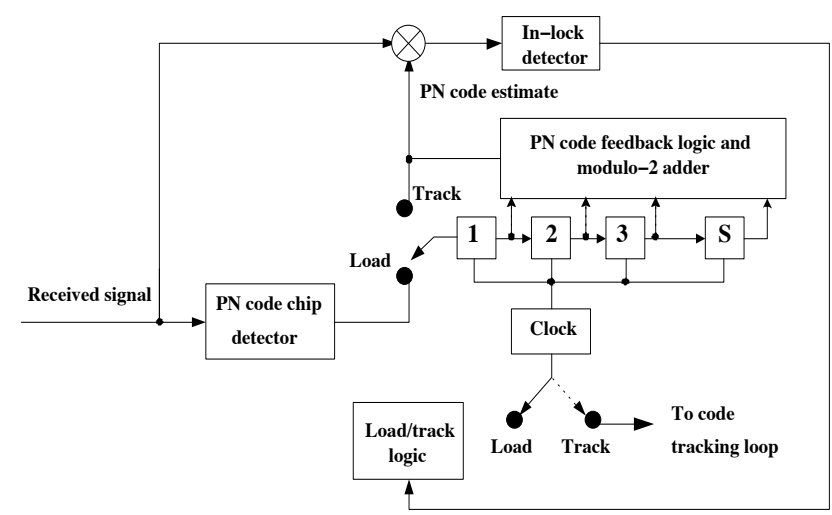

Fig. 6. Schematic diagram of the rapid acquisition using sequential estimation [12].

and of the widely known turbo principle [45]-[49] were proposed in [14], [50], [51]. Further details on these topics will be provided in the following subsections.

\section{3-1) Rapid Acquisition Using Sequential Estimation}

The concept of sequential estimation based code acquisition was originally proposed by Ward [12], where a sequential estimation of the Linear Feedback Shift Register (LFSR) states of the PN code sequence generator is employed. The schematic diagram of the RASE is provided in Figure 6. In this figure, the PN code chip detector estimates the first $S$ received PN code chips of the $\left(2^{S}-1\right)$-chip $m$-sequence, where $S$ is the number of LFSR stages uniquely and unambiguously describing the $m$-sequence. Then these chips are loaded into the LFSR based upon modulo-2 operations for the sake of generating the entire $m$-sequence having the correct codephase. The lock-detector determines, whether the related decision is deemed correct or not by using the various test statistics of [12] based upon the correlation between the received signal and the locally generated one. Finally, the decision concerning the activation of the reloading command or whether to activate the code tracking loop is controlled by the load/track logic module of Figure 6. Both the estimation and loading processes are performed periodically, until the correct initial LFSR state is successfully identified.

\section{3-2) Recursion-Aided Rapid Acquisition Using Sequential}

\section{Estimation}

The enhanced versions [13] of the original RASE schemes rapidly discard the majority of the incorrect code-phase estimation results and hence accelerate the search as well as increase the reliability of detecting the correct code-phase. Accordingly, this concept is similar to that of the multiple dwell based search [4], [13]. A modified version of the original RASE scheme [12], which is known as RARASE was proposed by Ward and Yiu [13]. This scheme exploits the fact that knowing $S$ consecutive chips of the PN sequence to be acquired uniquely and unambiguously describes all the $2^{S}-1$ chips of the $m$-sequence, because once the $S$ chips entered the shift-register seen in Figure 6, they determine all the $\left(2^{S}-1\right)$-chips. Hence based on the initial $S$ chips the receiver is capable of deciding whether the initial estimate of the entire $\left(2^{S}-1\right)$-chip received signal is correct or not. By invoking the procedure, a considerable proportion of lowlikelihood initial estimate may be discarded using a relatively 
simple device, which consists of several adders and an AND gate.

3-3) Majority Logic Decoding Based Rapid Acquisition Using Sequential Estimation

For the sake of generating the resultant $\left(2^{S}-1\right)$ chips, this scheme makes use of a majority voting method [52], which collects multiple independent estimates of the consecutive $S$ chips for the sake of obtaining the considerably higher number of up to $\left(2^{S-1}-1\right)$ parity-check sums and then employs a majority logic voting device for the sake of determining the initial 'S' chips to be loaded into the LFSR [44]. The number of multiple estimates is chosen based upon the achievable target performance, which typically varies from unity to $\left(2^{S-1}-1\right)$ [44], [52], [53]. Accordingly, the performance of this scheme is highly dependent upon the number of estimates used in the majority logic voting device. Compared to the RASE system of Figure 6, the correct detection probability of the scheme is considerably increased at the expense of an increased hardware complexity.

The detailed procedure of the MLD based rapid code acquisition procedure exploiting sequential estimation is as follows [44]. The incoming chip is shifted into the majority logic decoder. When all the $S_{\text {Load }}$ number of chips are fully loaded in the Decoder Shift Register (DSR), where the parameter $S_{\text {Load }}$ indicates the smallest span of chips required for the sake of evaluating a specific parity-check sum, a majority vote of the multiple estimates is taken and then the result is loaded into the local PN code generator as the estimate of the first initial load value. For the sake of obtaining all the estimates of the remaining chips, the above-mentioned process is repeated, until $S$ consecutive chips have been identified. Then the local PN code generator's outputs are correlated with the received codes over a specific examination period. During this time new chips are loaded into the majority logic decoder. If successful acquisition is not achieved at the end of the examination period, the next $S$ chips have to be determined by the decoder, where the $S$ chips are shifted into the local code generator, and then the same procedures are repeated again. The entire process is performed periodically, until the correct initial LFSR state is successfully identified.

\section{3-4) Recursive Soft Sequential Estimation}

The most imperative requirement for the sake of achieving successful acquisition of PN sequences based upon sequential estimation such as RASE [12] is that $S$ successive chips of the received noise contaminated PN sequence must be correctly estimated. Hence the iterative Soft-In/Soft-Out (SI/SO) decoding principle, may be invoked [48], which was inspired by turbo channel decoding [45]-[47], [49], [54], [55] in order to enhance the reliabilities associated with deciding on the $S$ consecutive chips. This RSSE acquisition scheme determining the $\left(2^{s}-1\right)$-chip $m$-sequence of Figure 7 exploits again the inherent properties of $m$-sequences uniquely and unambiguously determined by $S$ successive chips output by a recursive SI/SO decoder [14], [37], [50], [51], [56]. The recursive SI/SO decoder receives both soft information from the channel's output related to the current chip as well as soft extrinsic information [45]-[47], [49], [55] from the soft channel outputs related to the previous chips, which convey information dispersed by the channel encoder, but related to

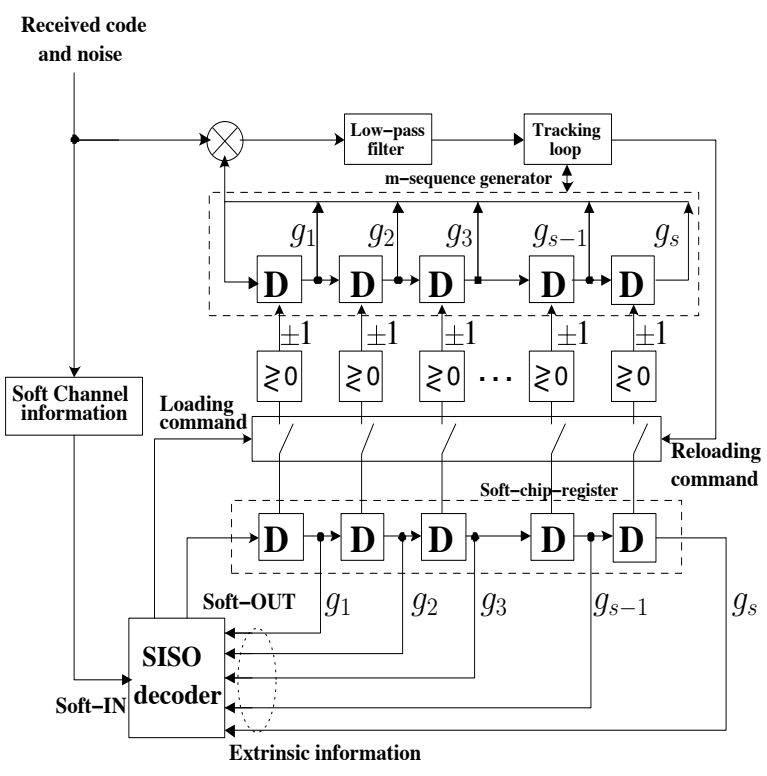

Fig. 7. Schematic diagram of the recursive soft sequential estimator [14].

the current chip. The SI/SO decoder then exploits both the soft channel-output information and the extrinsic information provided by the previous estimates of the SI/SO decoder in order to calculate a reliable soft output for the sake of updating the contents of the soft-chip register. The soft output of the recursive SI/SO decoder is then shifted into the so-called softchip register of Figure 7, which provides extrinsic information for supporting the forthcoming decoding steps. A cardinal characteristic of the RSSE acquisition scheme is that it makes use of the real-time knowledge of the reliabilities associated with the $S$ consecutive chips. The real-time knowledge of the chip reliabilities can be exploited for the sake of determining the probability of successful acquisition of an $m$-sequence of length $\left(2^{S}-1\right)$.

Again, the schematic diagram of the RSSE acquisition scheme is shown in Figure 7 [6], [14], which encompasses four constituent building blocks, namely, an $m$-sequence generator, a soft-chip register, a SI/SO decoder and a code phase-tracking loop. The soft-chip register has $S$ number of delay units, which are referred to as Soft-Chip Delay Units (SCDUs) of the $m$-sequence generator. The SCDUs store the instantaneous Log-Likelihood Ratio (LLR) values of $S$ consecutive chips. With the aid of these $S$ LLR values, $S$ consecutive chips can be determined and loaded into the corresponding delay units of the $m$-sequence generator of Figure 7. The SI/SO decoder estimates the corresponding LLR soft outputs after obtaining a soft channel output sample related to a given chip of the $m$-sequence. In addition to the so-called intrinsic information of this chip, which was received from the channel, the so-called a priori (extrinsic) information related to the chip, based upon the previous decoded LLR values stored in the SCDUs of Figure 7 is also exploited. The soft output of the SI/SO decoder is then shifted to the left-most position of the SCDUs in the soft-chip register, whilst the soft value in the right-most SCDUs is shifted out and dumped. More explicitly, the soft-chip register always stores the most recent $S$ number of soft outputs of the 
TABLE II

Contributions on Sequential Estimation Based Code ACQUISITION

\begin{tabular}{|c|c|c|}
\hline Year & Authors & Contribution \\
\hline 1965 & R. Ward [12] & $\begin{array}{l}\text { This scheme employs sequential estimation } \\
\text { of the LFSR states of the PN code sequence } \\
\text { generator. }\end{array}$ \\
\hline 1973 & C. Kilgus [44] & $\begin{array}{l}\text { This arrangement makes use of a majority } \\
\text { voting aided method, which collects } \\
\text { multiple independent estimation results for } \\
\text { each of ' } S \text { ' chips and then employs a } \\
\text { majority logic voting device for the sake } \\
\text { of determining the most reliable initial 'S' } \\
\text { chips to be loaded into the LFSR. }\end{array}$ \\
\hline 1977 & $\begin{array}{l}\text { R. Ward and Y. } \\
\text { Kai [13] }\end{array}$ & $\begin{array}{l}\text { This scheme rapidly discards the majority } \\
\text { of the incorrect estimation results, therefore } \\
\text { accelerates the search and also increases the } \\
\text { reliability of achieving correct acquisition. }\end{array}$ \\
\hline 1988 & $\begin{array}{l}\text { L.S. Lee and J.H. } \\
\text { Chiu [59] }\end{array}$ & $\begin{array}{l}\text { This sequential estimation based PN code } \\
\text { acquisition scheme employs a so-called } \\
\text { extended characteristic polynomial. }\end{array}$ \\
\hline 1994 & $\begin{array}{l}\text { R.T. Barghouthi } \\
\text { and G.L. Stüber } \\
\text { [60] }\end{array}$ & $\begin{array}{l}\text { A sequence acquisition scheme based } \\
\text { on both sequential estimation and soft- } \\
\text { decision combining methods was invoked } \\
\text { for DS-CDMA systems, which exploits the } \\
\text { algebraic properties of Kasami sequences } \\
\text { and hence becomes capable of providing a } \\
\text { very reliable estimate of the correct phase } \\
\text { of the local LFSR. }\end{array}$ \\
\hline 2002 & $\begin{array}{l}\text { L.L. Yang and L. } \\
\text { Hanzo [50] }\end{array}$ & $\begin{array}{l}\text { The proposed RSSE acquisition scheme } \\
\text { exploits the inherent properties of m- } \\
\text { sequences namely that they are uniquely } \\
\text { described by 'S' successive chips based } \\
\text { upon a recursive SI/SO decoder. }\end{array}$ \\
\hline 2004 & $\begin{array}{lrr}\text { J.H. } & \text { Lee, } & \text { I.H. } \\
\text { Song, } & \text { S.R. } & \text { Park } \\
\text { and } & \text { J.M. } & \text { Lee } \\
{[53]} & & \end{array}$ & $\begin{array}{l}\text { This scheme is a majority logic decoding } \\
\text { method based one using a decision logic } \\
\text { for the sake of improving the performance } \\
\text { of the majority logic decoding based RASE } \\
\text { of [44]. }\end{array}$ \\
\hline 2004 & $\begin{array}{l}\text { L.L. Yang and L. } \\
\text { Hanzo [14] }\end{array}$ & $\begin{array}{l}\text { The proposed RSSE acquisition scheme } \\
\text { exploits the inherent properties of m- } \\
\text { sequences namely that they are uniquely } \\
\text { described by 'S' successive chips based } \\
\text { upon a recursive SI/SO decoder. }\end{array}$ \\
\hline 2005 & $\begin{array}{l}\text { L.L. Yang and L. } \\
\text { Hanzo [51] }\end{array}$ & $\begin{array}{l}\text { This Differential Recursive Soft Sequential } \\
\text { Estimation (DRSSE) acquisition scheme } \\
\text { exploits the inherent properties of m- } \\
\text { sequences namely that they are uniquely } \\
\text { described by 'S' successive chips based } \\
\text { upon a recursive SI/SO decoder. }\end{array}$ \\
\hline 2005 & $\begin{array}{l}\text { K.M. Chugg and } \\
\text { M. Zhu [37] }\end{array}$ & $\begin{array}{l}\text { Iterative message passing algorithms are } \\
\text { applied to PN code acquisition problems } \\
\text { for the sake of approaching the achiev- } \\
\text { able maximum-likelihood synchronisation } \\
\text { performance. }\end{array}$ \\
\hline 2006 & $\begin{array}{l}\text { O. W. Yeung and } \\
\text { K.M. Chugg [56] }\end{array}$ & $\begin{array}{l}\text { A new iterative algorithm and its hardware } \\
\text { architecture are proposed based on iterative } \\
\text { message passing algorithms which are } \\
\text { applied in the context of an ultra-wideband } \\
\text { system. }\end{array}$ \\
\hline
\end{tabular}

SI/SO decoder, which corresponds to $S$ consecutive chips of the transmitted $m$-sequence.

Whenever the amplitude of the most recent $S$ soft outputs of the SI/SO decoder becomes sufficiently high for the sake of guaranteeing a sufficiently low erroneous loading probability, a "loading command" is activated. Then $S$ consecutive chips are decided, employing hard decisions based upon the most recent $S$ LLR values stored in the soft-chip register of Figure 7. Then, the $S$ successive chips are loaded into the corresponding delay units of the local $m$-sequence generator. Once the $m$-sequence generator is loaded with the initial chip values, the received spread-spectrum signal can be despread, employing the locally generated $m$-sequence replica, provided that the initial chip values of the $m$-sequence generator have been correctly loaded. The despread signal is then low-pass filtered and sent to the code tracking loop. If the code tracking loop is capable of tracking the phase, the entire code acquisition process is completed. By contrast, unless the tracking loop is capable of tracking the phase, the code tracking loop activates a "reloading command" in an effort to load another group of $S$ successive chips into the delay units of the $m$-sequence generator. The above process will be repeated, until code acquisition is successfully accomplished. Finally, the total mean acquisition time of the RSSE scheme can be approximately determined by the duration of time required for the RSSE to accomplish recursive SI/SO decoding, so as to achieve a sufficiently low erroneous loading probability. Table II shows the history of a variety of sequential estimation based code acquisition schemes and the evolution of sequential estimation in spread-spectrum communication systems.

4) Random Search Based Code Acquisition: When considering a realistic UWB channel, the numerical analysis of serial search based schemes becomes intractable for the channel impulse response constituted by sparse clumps of multi-path components [57]. However, recently a random search aided scheme was proposed as a realistic alternative for the analysis of the UWB channel model [58], because the random search stipulates no particular assumption regarding the channel model and hence can be applied to arbitrary models. Furthermore, based on the results of Fig.6 in [58], the performance of the serial search based scheme approaches that of the random search. The schematic of the random search aided receiver is exactly the same as that of the serial search based one seen in Figure 4, except that the search algorithm shifts the code phase of the local sequence by a random amount selected between 0 and $(\nu-1)$, where $\nu$ represents the number of chips in the entire uncertainty region to be searched.

\section{B. Detector Structure}

1) Non-Coherent Code Acquisition: Initial code acquisition is incapable of employing any prior information concerning the transmitter's carrier phase. Therefore, during initial code acquisition either NC [3]-[6] or DC [18], [19] schemes have to be used. The system seen in Figure 8 explicitly shows an example of the NC scheme's structure. Observe in Figure 8 that the chip energy accumulated during a predetermined integration dwell time is squared and then the accumulated energies of both the I and Q branches are summed in order to generate a final decision variable.

2) Differentially Coherent Code Acquisition: Similarly to the NC code acquisition scheme of Figure 8, DC code acquisition [18] does not require any prior information concerning the transmitter's carrier phase. An additional benefit of employing the DC code acquisition scheme of Figure 9 is that it is capable of providing a better performance than using a NC one [18], [19]. This is, because the DC scheme 


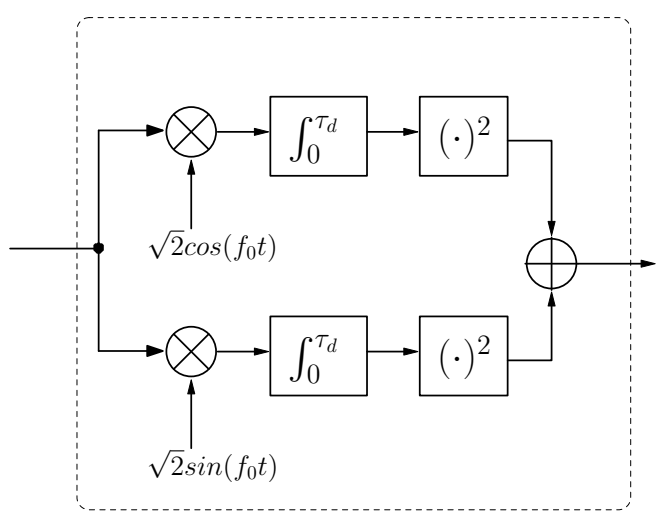

Fig. 8. Schematic diagram of the non-coherent receiver.

has a performance gain of just under $3 \mathrm{~dB}$ in comparison to the $\mathrm{NC}$ one in terms of a pair of the correct detection and false alarm probabilities. There are two types of DC code acquisition schemes in the literature [17], [18]. First of all, Chung [17] proposed the chip-based DC detection scheme of Figure 9 that employs a differential receiver having a one-chip time delay, which has the potential of mitigating the typical code acquisition performance degradation experienced in fast-fading mobile channels, where $T_{c}$ is the chip duration, the integral dwell time represents $\tau_{D}, c(t)$ denotes the unique user-specific PN sequence and $f_{0}$ is the carrier frequency. The chip based-DC receiver of Figure 9 first evaluates the modulo-2 function of the current and previous chip values for both the received $m$-sequence and the locally generated $m$ sequence. As mentioned before, at this stage no knowledge of the transmitter's carrier phase is available at the receiver, but it is reasonable to assume that the channel-induced magnitude and phase changes of two consecutive chips are similar. Hence, by exploiting this assumption, the differential detector of Figure 9 eliminates the effects of the channel-induced as well as carrier-drift-induced phase changes and despite the absence of a coherent detector, it has a good chance of correctly detecting the transmitted $m$-sequence. Then, as seen in Figure 9, the differentially encoded received as well as locally stored $m$-sequences are compared, i.e. correlated with each other and the correlator output is compared to the decision threshold of $\theta$. As also shown in Figure 9, there are two hypotheses, namely $H_{1}$ and $H_{0}$, which correspond to the desired signal being either present or absent, respectively. For further details please refer to [17].

Secondly, instead of squaring the energy accumulated over $N$ chips as suggested by the principle of the $\mathrm{NC}$ receiver of Figure 8, the channel's chip-based output samples are summed over either fraction of or over the entire duration of a full $m$-chip spreading code period, which are then multiplied by the conjugate of the $N$-chip-delayed samples [18] ${ }^{5}$, according to the philosophy of the Partial-PeriodCorrelation (PPC) [18] or the Full-Period Correlation [18]

\footnotetext{
${ }^{5}$ Selection of the optimised $N$ value is highly dependent upon the performance degradation of the carrier frequency mismatch between the BS's transmitter and the MS's receiver.
}

(FPC) based schemes ${ }^{6}$, respectively. The schematic diagram of the DC code acquisition based receiver employing FPC is shown in Figure 10. It is interesting to note in [18] that the achievable detection performance of the partial and full correlation based methods becomes similar in the context of $\mathrm{NC}$ acquisition schemes, as the length of the integration used by the correlation of Figure 8 is gradually increased, whilst their performances are rather different in the context of DC schemes [18]. The superiority of the DC schemes is a consequence of the statistical characteristics of the noise samples contaminating the successive channel output samples in the low SINR region. Namely, the DC scheme is more efficient in terms of reducing the effects of AWGN and interference than the NC one in the low SINR ranges. To elaborate on the above fact a little further, in the low SINR region, the false alarm probabilities of the DC and NC schemes differ by a factor of two. This result leads to the superiority of the DC scheme over the NC one [18]. DC schemes outperform the corresponding $\mathrm{NC}$ arrangements in terms of both their correct detection probability and false alarm probability ${ }^{7}$, and hence they also exhibit a considerably better achievable MAT performance. Table III outlines the history of DC code acquisition schemes.

3) Post-Detection Integration: The PDI technique of Figure 11 [3], [15], [16] is widely used in initial code acquisition and/or post-initial code acquisition modules of the commercial CDMA based systems, such as the CDMA-2000 [1] and W-CDMA systems [2]. The underlying philosophy is that a decision variable is generated by accumulating $T$ consecutive $\mathrm{N}$-spaced signal samples observed over multiple $\mathrm{N}$-spaced time intervals to improve the correct detection probability in the mobile channel imposing both fading and poor SINR conditions. In order to improve the correct detection probability at the cost of a minor hardware increase compared to the complexity of acquisition modules using no PDI, more explicitly, the number of PDI stages must be increased, requiring the summation of a specific number of consecutive N-chip-spaced integrator outputs for the sake of generating the final decision statistics ${ }^{8}$. The number of PDI stages should be determined depending on whether initial code acquisition or post-initial code acquisition is considered, as well as depending on the number of both transmit and receive antennas for the sake of achieving the minimum required code acquisition performance. There is a tradeoff between the number of the PDI stages and the attainable MAT performance. Figure 11 depicts the schematic of a serial search based receiver employing the PDI technique of [3], [15], [16].

\footnotetext{
${ }^{6}$ The main philosophical difference of the PPC and FPC techniques is that the correlation is performed over a predefined segment of the total PN code sequence in conjunction with PPC, whereas the correlation is carried out over a full $m$-chip spreading code period when exploiting FPC.

${ }^{7}$ The false alarm probability is defined as the probability of mistakenly deciding on which specific spreading sequence was used at the desired user's transmitter, which is a direct consequence of the channel-induced impairments imposed by fading, noise and inteference.

${ }^{8}$ This specific number must be determined by satisfying a pair of targeted correct detection and false alarm probabilities in terms of minimised MAT.
} 


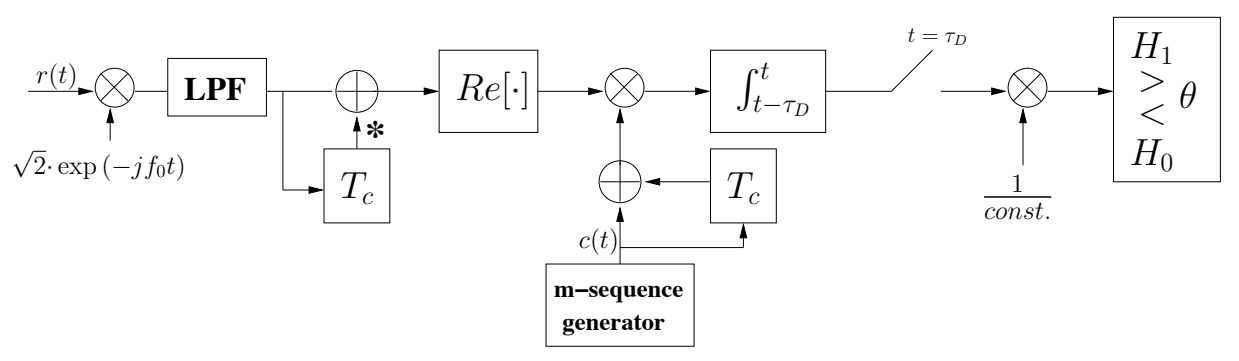

Fig. 9. Schematic diagram of the chip based-differentially-coherent acquisition scheme [17].

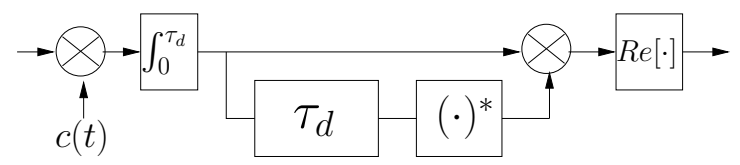

Fig. 10. Schematic diagram of the differentially-coherent acquisition based receiver employing FPC.

\section{Initial and Post-Initial Code Acquisition}

The classic serial search techniques designed for initial code acquisition [3] have been traditionally employed in specific scenarios, where the uncertainty region (or search window width) is quite wide (i.e. $2^{15}-1$ ) and hence in the context of serial search it is the MAT, which constitutes the most pertinent performance criterion, as seen for example in the DL of the inter-cell synchronous CDMA-2000 system [3]. In the case of initial code acquisition contrived for DS-CDMA, the main design goal is to acquire accurate timing of the first received signal path impinging at the receiver, since this timing information is used as that of the reference finger of the Rake receiver. By contrast, the post-initial code acquisition procedure that extracts the accurate timing positions of the remaining delayed paths and identifies the appropriate paths earmarked for processing by the Maximum Ratio Combining (MRC) scheme of the Rake receiver, has a major impact on the performance of the Rake receiver [8]. There are two main differences between the initial and post-initial code acquisition procedures. First of all, once the first Rake finger is synchronised, the uncertainty region that has to be explored will be shrunk to $\pm \xi$ hypotheses surrounding the timeinstant, where the first received path was found. This reduced interval will be referred to as the 'reduced uncertainty region' to be explored after the initial code acquisition [7]. This search window width is defined by both the dispersion of the multipath propagation environment encountered as well as by the appearance and disappearance of propagation paths [66]. Secondly, the post-initial code acquisition procedure commences after the AFC operation was activated for the sake of fine tracking, following the successful initial code acquisition. Hence, the performance degradation imposed by the associated frequency mismatch is considerably reduced compared to that immediately after the initial code acquisition.

\section{Code Acquisition in MC-DS-CDMA}

During the early 1990s, MC-DS-CDMA transmission schemes have been proposed in [67], in order to achieve further advantages in terms of bandwidth efficiency, frequency

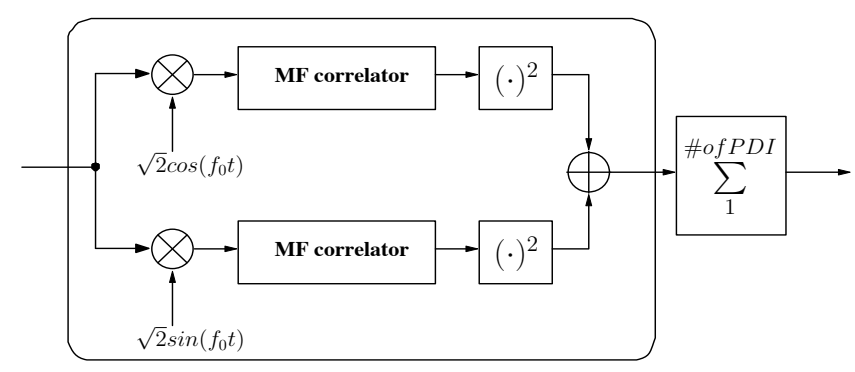

Fig. 11. Schematic diagram of the serial search based receiver employing post-detection integration.

diversity, reduced-complexity parallel signal processing and interference rejection capability in high data-rate transmissions [68]-[70]. The parallel code acquisition performance of a MC-DS-CDMA system has been analysed in [21], when communicating over AWGN or flat Rayleigh fading channels. In the MC-DS-CDMA system considered in [21], the authors characterised various code acuqisition scenarios in the context of a MC-DS-CDMA system based upon both Equal Gain Combining (EGC) and Selection Combining (SeC). The performance of both scenarios was examined in both AWGN and Rayleigh fading channels. To elaborate a little further, it was stated in [21] that the performance of using EGC is better than that when exploiting $\mathrm{SeC}$ in the context of an MC-DS-CDMA or single-carrier system. These conclusions were applicable to the UL case, i.e. the MS to BS link of both MC- and SC- DS-CDMA systems. A parallel search based maximum-likelihood code acquisition scheme was adopted at the receiver. On the other hand, the code acquisition performance of MC-DS-CDMA attained with the aid of a serial search based scheme has been investigated in [6], [20].

Figure 12 illustrates the schematic diagram of the transmitter used in the generalised MC-DS-CDMA system of [20], where $u=1, \ldots U$ denotes the number of subcarriers, $c(t)$ is the unique user-specific PN sequence, $f_{u}$ denotes the $u^{t h}$ subcarrier frequency and $\phi_{u}$ is the $u^{t h}$ subcarrier phase of the modulator. Furthermore, $T_{b}$ indicates the bit duration of the data sequence before Serial-to-Parallel (SP) conversion, whilst $T_{s}$ represents the symbol duration after SP conversion. In the MC-DS-CDMA system considered the input bit sequence is SP converted and each of the parallel sequences is then transmitted on a separate subcarrier. Consequently, we have $T_{s}=U \cdot T_{b}$. Furthermore, $S F=T_{s} / T_{c}$ denotes the spreading factor of the subcarrier signals in the MC-DS-CDMA system, whilst the spreading factor of a corresponding identicalbandwidth SC-DS-CDMA system is $S F_{1}=T_{b} / T_{c 1}$, where 
TABLE III

Contributions on Differentially CoHerent Code Acouisition

\begin{tabular}{|c|c|c|}
\hline Year & Authors & Contribution \\
\hline 1994 & $\begin{array}{l}\text { H.S. Liaw and } \\
\text { C.D. Chung [61] }\end{array}$ & $\begin{array}{l}\text { A chip based DC detection scheme was } \\
\text { proposed, which employs a differential } \\
\text { receiver having one-chip time delay in } \\
\text { an effort to mitigate the code acquisition } \\
\text { performance degradation inflicted by fast } \\
\text { fading mobile channels. }\end{array}$ \\
\hline 1995 & C.D. Chung [17] & $\begin{array}{l}\text { A chip based DC detection scheme was } \\
\text { proposed, which employs a differential } \\
\text { receiver having one-chip time delay in } \\
\text { an effort to mitigate the code acqui- } \\
\text { sition performance degradation inflicted } \\
\text { by high-Doppler fading mobile channels. } \\
\text { The proposed serial search based code } \\
\text { acquisition scheme using chip based DC } \\
\text { detection outperforms its parallel search } \\
\text { based counterpart in terms of the achievable } \\
\text { mean acquisition time. }\end{array}$ \\
\hline 1997 & $\begin{array}{l}\text { M.H. } \\
\text { Zarrabizadeh, } \\
\text { and E.S. Sousa } \\
{[18]}\end{array}$ & $\begin{array}{l}\text { In this scheme, the channel's output } \\
\text { samples are summed over either a fraction } \\
\text { or the entire duration of a full spreading } \\
\text { code period and they are multiplied by } \\
\text { the conjugate of the } \tau_{D} \text {-chip-delayed } \\
\text { samples for the sake of achieving a } \\
\text { better performance over the noncoherent } \\
\text { counterpart, as seen in Figure } 10 \text {. }\end{array}$ \\
\hline 2001 & $\begin{array}{ll}\text { T. } & \text { Ristaniemi } \\
\text { and } & \text { J. Joutsen- } \\
\text { salo } & {[62]}\end{array}$ & $\begin{array}{l}\text { Coarse delay estimates are obtained by } \\
\text { both a Differential Correlation based } \\
\text { Matched Filter (DC-MF) and a Differential } \\
\text { Correlation based MUlti-class SIgnal Clas- } \\
\text { sifier (DC-MUSIC) in order to efficiently } \\
\text { mitigate the effects of both noise and } \\
\text { interferers. }\end{array}$ \\
\hline 2002 & $\begin{array}{l}\text { Y.K. Jeong, O.S. } \\
\text { Shin and K.B. } \\
\text { Lee [63] }\end{array}$ & $\begin{array}{l}\text { Slot synchronisation designed for fast and } \\
\text { reliable cell search was proposed for } \\
\text { inter-cell asynchronous DS-CDMA systems } \\
\text { employing a DC combining scheme, using } \\
\text { multiple observations over a number of } \\
\text { slots in order to increase the reliability of } \\
\text { code acquisition. }\end{array}$ \\
\hline 2002 & $\begin{array}{lll}\text { J.C. } & \text { Lin } \\
{[64]} & & \end{array}$ & $\begin{array}{l}\text { DC PN code acquisition using FPC in both } \\
\text { chip-synchronous and chip-asynchronous } \\
\text { direct sequence/spread spectrum receivers } \\
\text { was designed and analyzed. }\end{array}$ \\
\hline 2003 & $\begin{array}{l}\text { O.S. Shin and } \\
\text { K.B. Lee [65] }\end{array}$ & $\begin{array}{l}\text { The employment of DC combining was } \\
\text { proposed for improving the performance } \\
\text { of a double-dwell acquisition system by } \\
\text { increasing the reliability of the code-phase } \\
\text { verification stage. }\end{array}$ \\
\hline 2005 & $\begin{array}{l}\text { L.L. Yang and L. } \\
\text { Hanzo [51] }\end{array}$ & $\begin{array}{l}\text { The proposed Differential Recursive Soft } \\
\text { Sequential Estimation (DRSSE) acquisition } \\
\text { scheme exploits the inherent properties of } \\
\text { m-sequence estimates, namely that they are } \\
\text { uniquely and unambiguously described by } \\
\text { 'S' successive chips based upon a recursive } \\
\text { SI/SO decoder invoking differential pre- } \\
\text { processing. }\end{array}$ \\
\hline
\end{tabular}

$T_{c 1}$ represents the chip duration of the corresponding SC-DSCDMA signal. For the sake of simplicity, in our forthcoming discourse we assume that there is no overlap between the main spectral lobes of two adjacent subcarriers in the MCDS-CDMA system considered [20]. Furthermore, we postulate that each subcarrier signal occupies an identical bandwidth and the total bandwidth is equally divided among the $U$ number of subcarriers. Hence, the relationships of $T_{c}=U \cdot T_{c 1}$ and $S F_{1}=S F$ hold, since we have $T_{s}=U \cdot T_{b}$. Based upon the above assumptions, both the MC- and the corresponding SC-DS-CDMA systems maintain an identical bandwidth of

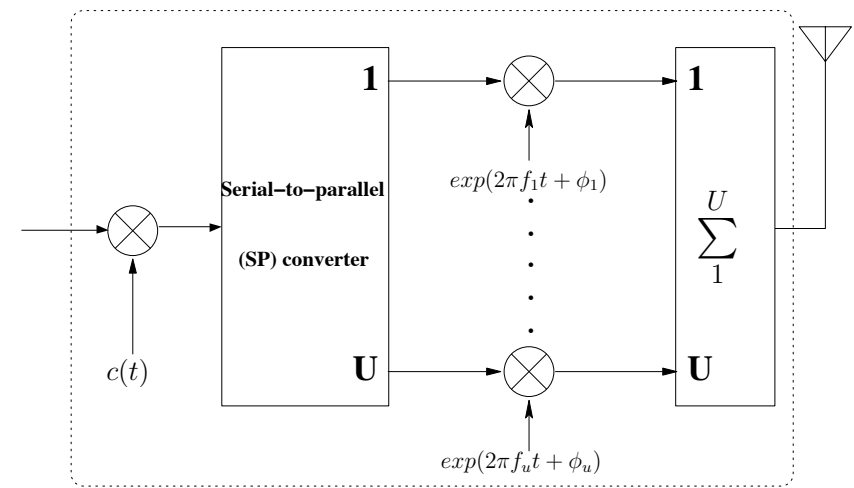

Fig. 12. Transmitter schematic of the MC-DS-CDMA downlink having $U$ subcarriers.

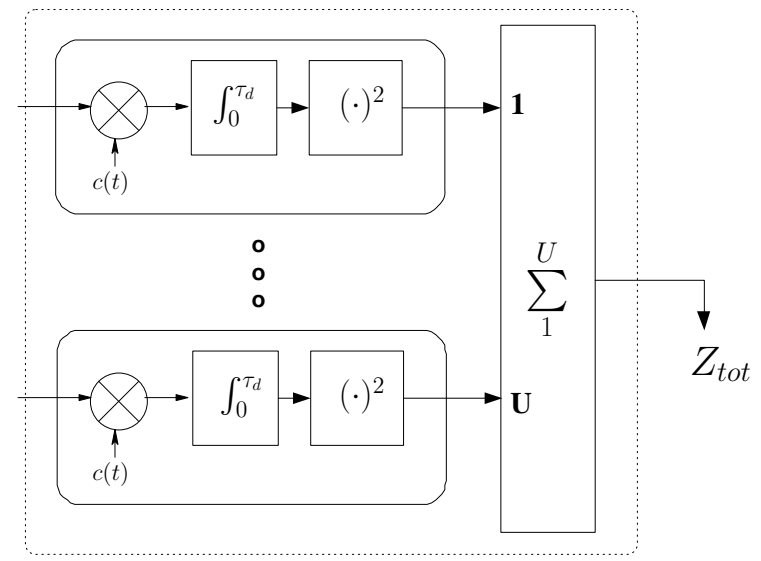

Fig. 13. Receiver structure of a noncoherent code acquisition system employing $U$ subcarriers.

$2 / T_{c 1}$, as proposed in [20]. It is also worth noting that the PN sequence' chip duration $T_{c 1}$ of the SC-DS-CDMA system is $U$ times lower than that of the MC-DS-CDMA arrangement. Hence we have $T_{c 1}=T_{c} / U$. This is because given the same allocated bandwidth and the same total transmitted energy per chip, the bandwidth of the SC-DS-CDMA signal is $U$ times higher than that of the subcarrier signals in the MC-DSCDMA system using $U$ subcarriers. Moreover, in an effort to maintain a constant integral dwell time of $\tau_{D}$, the chip energy summed by the SC-DS-CDMA receiver during the period of $\tau_{D}$ is $U$ times higher than that collected by the MCDS-CDMA correlator of each subcarrier, since the number of chips within the period of $\tau_{D}$ is $U$ times higher for the SCDS-CDMA system than that of the MC-DS-CDMA system [20]. The receiver structure of a serial search based NC code acquisition scheme employing $U$ subcarriers is portrayed in Figure 13. The received signal is first down-converted to each subcarrier. For each subcarrier the corresponding correlator evaluates the correlation between the locally generated PN sequence and the received baseband signal, while integration takes place over the duration of the dwell time of $\tau_{D}$ seconds. Then the output of each correlator is squared and accumulated. Finally, the outputs of the $U$ NC subcarrier correlators are combined, in order to generate the output variable, $Z_{\text {tot }}$ seen in Figure 13. Observe in Figure 6 (a) of [20] that the code acquisition performance of a MC-DS-CDMA system using 
EGC significantly improves, as the number of subcarrier signals combined increases, over the SINR per chip $\left(E_{c} / I_{0}\right)$ range considered. Moreover, the code acquisition performance of the MC-DS-CDMA system employing EGC was formed to be better in Figure 6 of [20] than that of the corresponding MC-DS-CDMA system employing SeC and also better than that of the identical bandwidth SC-DS-CDMA system. The major trend is that the characteristics of the serial search based code acquisition arrangements are similar to those of the parallel search based one.

\section{CODE ACQUISITION IN DS-UWB}

\section{A. Overview of Code Acquisition Schemes}

The research of UWB systems has recently attracted a significant interest in both the academic and industrial community [34], [35]. The emerging UWB systems are capable of supporting both wireless personal computers and home entertainment equipment, both requiring high data rates. Similarly, UWB systems may also be used in a variety of sensor networks operating at low data rates and at a low power consumption. DS-UWB techniques are characterised by low-duty-cycle pulse trains having a very short impulse duration [36], [37], because the high bandwidth results in a fine resolution of the timing uncertainty region. Depending on the logical value to be conveyed, a signalling impulse of $T_{p}$ width having the required polarity is allocated at multiples of the frame duration $T_{f}$, where $T_{f}$ is defined as the pulse repetition period, i.e. the time between two consecutive signalling pulses. In the DS-UWB DL, initial acquisition is required for both coarse timing as well as for code phase alignment and both of these constitute a challenging problem owing to the extremely short chip-duration [36], [37]. Again, this leads to a huge search space size, which is represented as the product of the number of legitimate code phases in the uncertainty region of the PN code and the number of legitimate signalling pulse positions. Both the Timing Acquisition (TA) and PN Code Phase Acquisition (CPA) must be achieved within the allowable time limits. Most acquisition schemes considered in the literature rely on either serial- or hybridsearch based acquisition schemes [36], [58], [71]. Relatively short PN codes have to be employed, in order to avoid having an excessive search space.

The code acquisition schemes typically used in the DS-UWB DL may be categorised into the following five classes:

1) Detection based approaches: [36]

Some of the acquisition schemes proposed for the DS-UWB DL are reminiscent of those applied in conventional DS-CDMA systems.

2) Structured search strategies: [36], [58]

A search strategy specifies the order in which the candidate phases in the timing uncertainty region are evaluated by the acquisition scheme. When there are more than one acquisition phases in the uncertainty region, the serial search which simply searches through the uncertainty region in a consecutive manner is no longer the optimal search strategy. More efficient non-consecutive search strategies, such as for example the 'look-and-jump-by-KK-bins' search and the bit-reversal search were proposed in [36]. For the scenario, when the acquisition phases to be evaluated are KK consecutive phases in the uncertainty region, it has been claimed that the 'look-and-jump-by-KK-bins' search is the optimal serial search permutation when $\mathrm{KK}$ is known, while the 'bit-reversal' is the optimal search permutation, when KK is unknown. Moreover, the random search may also be considered to be an efficient search strategy, which relies on the specific characteristics of the UWB channel model [58].

3) Search space reduction techniques: [36], [71]-[73] Some of the acquisition techniques have been dedicated to the reduction of the search space by using two-stage acquisition schemes. More specifically, a two-stage acquisition scheme obeying a specific signalling format has been characterised in [36], [72] and as a result, the size of the search space has been reduced to a certain degree.

4) Estimation-based schemes: [36]

Certain solutions designed for code acquisition in DS-UWB systems have employed estimation theoretic methods to attain the timing information of the received signal.

5) Iterative MP algorithm based schemes: [6], [14]

A variety of sequential estimation based code acquisition schemes have been proposed in the literature [5], [74]. As another attractive design option, recursive soft sequence estimation aided acquisition based on the iterative soft-in soft-out decoding principle has also been proposed in [6], [14]. These iterative acquisition schemes have been designed for PN codes by exploiting the available a priori knowledge about how PN codes are generated with the aid of LFSRs. Explicitly, a $\left(2^{S}-1\right)$-chip PN code can be generated with the aid of a LFSR using a specific Primitive Polynomial (PP), once the associated $S$-stage LFSR was filled with $S$ number of chip values [56], [75]. This beneficial property can also be exploited by the initial acquisition scheme at the receiver, because once we estimated $S$ number of channel-contaminated chip values, the acquisition scheme becomes capable of reconstructing the entire $\left(2^{S}-1\right)$-chip code. Recently, in [37], [56] the authors proposed rapid code acquisition schemes based on the iterative MP algorithm, which adopted a philosophy similar to that used for Low Density Parity Check (LDPC) codes. When considering high-reliability military systems, where the employment of long PN codes is necessary for achieving robustness against malicious jamming and interception, the schemes of [37], [56], [76], [77] are beneficial in terms of reducing the size of the search space. Further details on these topics will be provided in the following subsections. 


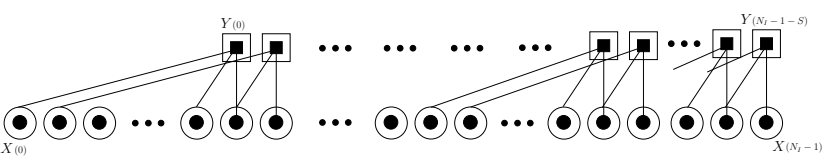

Fig. 14. Tanner graph based acquisition structure for the PN sequence employing the GP of $g_{1}(D)=D^{15}+D+1$. The squares and circles denote CNs and VNs, respectively.

\section{B. Iterative MP Aided Code Acquisition Schemes}

In the field of engineering, many problems have been solved by 'divide-and-conquer' based approach. Similarly, a variety of problems may be solved by using MP algorithms, where the messages are passed back and forth among simple processors. After a sufficiently high number of information exchange steps we may approach the global solution of a problem. In this spirit, this MP algorithm requires amongst the processing nodes, which carry out simple operations such as storage and addition of integers. A popular graphical model derived for characterising diverse probability distributions is constituted by the Tanner graph [78]. The Tanner graph is a bipartite graph, which characterises the relationship of the symbols of a codeword and the code constraints for the sake of constructing a valid codeword. A factor graph is a straightforward generalisation of the Tanner graph [79]. The factor graph is also a bipartite graph, which has a Variable Node (VN) for each variable and a Check Node $(\mathrm{CN})$ for describing the corresponding relationship among these VNs. In probabilistic modelling of arbitrary systems, the factor graph may be used for representing the joint probability density of variables describing the system. Factorisation of this joint probability density function into individual density functions encompasses vital information regarding the statistical dependencies among these variables. The calculation of a conditional probability corresponds to the so-called marginalisation of the related messages entered to each node [80]. The graphical model seen in Figures 14 and 15 can also be used for visualising the Parity-Check (P-C) constraints represented by connecting the VNs to the appropriate CNs. The simplest possible graphical model is based on a single $\mathrm{CN}$, which checks the parity of the specific binary variables connected to it. When considering a MP algorithm that repeatedly passes messages across the Tanner graph's edges in both directions, the MP algorithm merges and marginalises the messages related to the VNs by taking into account the constraints imposed by the CNs. Similarly, each $\mathrm{CN}$ will collect soft-decision information from the VNs connected to it. This soft information gleaned from the VNs connected to a $\mathrm{CN}$ is then combined in order to generate soft-decision based estimates, which are then subjected to a hard-decision, once the affordable number of iterations has been exhausted.

Figure 14 depicts the schematic designed for PN sequences generated using the Generator Polynomial (GP) of $g_{1}(D)=$ $D^{15}+D+1$ [37], [56], whilst Figure 15 portrays the schematic designed for PN sequences generated using the GPs of $g_{1}(D)=D^{15}+D+1$ and $g_{3}(D)=D^{60}+D^{4}+1[56]$, where the squares and circles represent $\mathrm{CNs}$ and VNs, respectively. Each $\mathrm{CN}$ in Figures 14 and 15 gleans soft-decision information

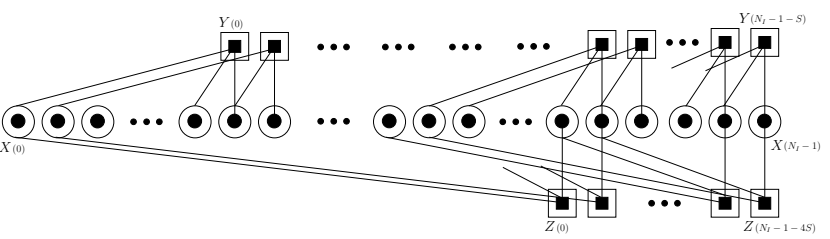

Fig. 15. Tanner graph based acquisition structure for the PN sequence employing the GPs of $g_{1}(D)=D^{15}+D+1$ and $g_{3}(D)=D^{60}+D^{4}+1$. The squares and circles denote CNs and VNs, respectively.

from the three VNs connected to it. This constraint obeys the structure of $g_{1}(D)$ as well as either $g_{1}(D)$ or $g_{3}(D)$ for Figures 14 and 15, respectively. To elaborate a little further, in case of $g_{1}(D)$, the first $\mathrm{CN} Y_{(0)}$ is directly connected to $X_{(0)}, X_{(14)}$ and $X_{(15)}$, corresponding to the terms of $D^{15}, D$ and 1 , respectively. Similarly, in Figure $15 Z_{(0)}$ associated with $g_{3}(D)$ is mapped into $X_{(0)}, X_{(56)}$ and $X_{(60)}$, corresponding to the terms of $D^{60}, D^{4}$ and 1 , respectively. Moreover, each node seen in Figures 14 and 15 represents a random variable, whereas each edge indicates probabilistic constraints among the nodes connected to it. The procedure of generating a $\left(2^{S}-1\right)$-chip PN sequence imposing redundancy according to the above-mentioned GPs may be considered to be equivalent to incorporating redundant $\mathrm{P}-\mathrm{Cs}$ into the standard Parity Check Matrix (PCM) of classic LDPC codes and a similar technique has been applied also for the soft decoding of classic channel codes in [81]. Each of the subgraphs corresponding to connections of the upper and the lower half of Figure 15 is based on a different GP, namely on $g_{1}(D)$ and $g_{3}(D)$, respectively. Mathematically, different reducible GPs may be used to generate the same PN sequence [56], [81]. We define the initial soft-decision based estimate in the form of $X(j)$ at the $\mathrm{VN}$, where $X(j)$ represents an $j^{t h}$ chip-estimate of an $N_{I}$-chip soft-sequence received, where we have $j=0, \ldots,\left(N_{I}-1\right)$. The set of $N_{I}$-chip received signal estimates becomes the initial input of all the related CNs. Figure 15 can be also interpreted as a PCM in case of employing $1^{\text {st }}$ and $3^{r d}$ order GPs.

\section{Two-Stage Iterative Acquisition}

Figure 16 portrays the transmitted UWB signal designed for two-stage acquisition, namely for the TA and CPA stages [36], [72]. The specifically designed training signal transmitted during the acquisition process is constituted by the superposition of the signals designed for supporting TA and CPA stages. Observe in Figure 16 that the top trace indicates a separate periodic pulse train used for supporting the TA stage, whilst the middle trace portrays a DS pulse train employed for assisting the CPA stage.

The schematic of the proposed receiver designed for Single Input Multiple Output (SIMO) scenarios is portrayed in Figure 17, respectively. These schemes are constituted by the amalgam of the sliding correlator used for the TA stage and the iterative CPA decoder, where $T_{T A}$ is a threshold value assigned to the TA stage and $T_{C P A}$ is another threshold assigned to the verification mode of the CPA stage [56], [72]. More specifically, the timing of the periodic pulse train at the TA stage is recovered by correlating the received signal 


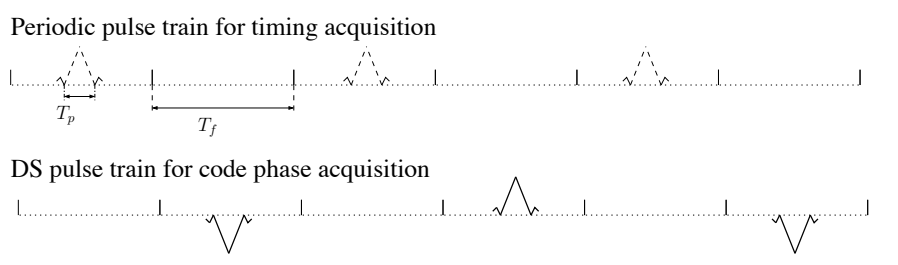

Transmitted signal for two-stage acquisition

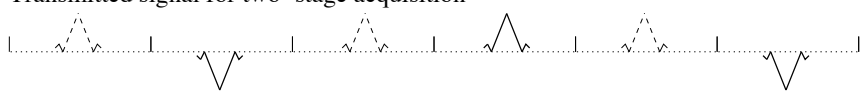

Fig. 16. The transmitted UWB signal designed for two-stage acquisition, namely for TA and CPA stages.

with the receiver's own replica of the periodic pulse train over the entire uncertainty region, which is twice $T_{f}$ [36], [72] and then comparing the correlator's output to the decision threshold of $T_{T A}$. More explicitly, we consider the random search technique at the TA stage. Once the TA stage is completed, the chip boundaries of the DS pulse train have become known and the CPA stage has to search for the correct phase across a single PN sequence duration. The employment of both the MP decoding algorithm [37] originally derived for LDPC codes and a single correlation required for the verification of the $\left(2^{S}-S-1\right)$ chips' expected values based on the $S$ consecutive chips hypothesised are considered.

\section{Performance Versus Complexity Associated}

Before moving on to the next section, for the sake of clarifying the behaviour of a variety of acquisition schemes, we provide a simple analysis. In order to simplify our comparison, when assuming the most optimistic scenario, the detection probability is set to ' 0 '. We choose a PN sequence defined by $g(D)=1+D+D^{r}$, where $r=15$. It is postulated that the entire length of the PN code is set to $2^{r}-1$, where $r=15$. The number of chips accumulated $(N)$ is 256 . Then, a hybrid detector employing eight parallel correlators is considered, where $T_{c}$ represents the chip duration of the PN sequence. Based on the above arguments and on the assumptions portrayed in [37], we characterise the relationship among the MAT, the memory requirements and the computational complexity of the serial, hybrid, parallel and iterative MP based acquisition schemes. Table II summarises the associated results. The values in parenthesis represent results calculated by exploiting the aforementioned parameters. Further detialed assumptions and formulations may be found in [37].

\section{CODE ACQUisition for Antenna ARrays as Well AS FOR CO-LOCATED AND COOPERATIVE MIMOS}

\section{A. Code Acquisition for Antenna Arrays}

In BeamForming (BF)-aided receivers so-called 'angular' division multiple access can be performed. Given an antenna array having $N$ elements, a $\mathrm{BF}$ scheme is capable of generating a set of $N$ spatially orthogonal(non-overlapping) beams in the required angular directions. The following code acquisition approaches are based on the characteristics of $\mathrm{BF}$ schemes. Below we continue by providing a summary of code acquisition schemes designed for antenna arrays [23][27]. In [23], code acquisition exploiting a multidimensional maximum likelihood delay estimator was investigated in the context of an adaptive antenna array designed for an unknown channel, where the interference had either known or unknown covariance. In [24], the conventional serial search based code acquisition operating in the delay-domain was extended to a two-dimensional (2-D) method, where the acquisition was performed in terms of both the delay-domain and in the angular domain. This scheme was conceived for investigating the potential benefits of compact antenna arrays having closely placed elements. However, when considering high interference peaks, the employment of 2-D code acquisition may no longer be attractive compared to the coventinal serial search operating in the delay-domain. By contrast, the 2-D search may become beneficial and may outperform the conventional one if a suitable number of angular partitions is selected. As a further advance, it was suggested in [25] that a 2-D code acquisition scheme using adaptive antenna arrays and least mean square optimization is capable of achieving an improved MAT performance - naturally, at the cost of an increased complexity. The classic acquisition techniques operating in the time-delay domain were then also extended to the angular domain by Puska et al. [26]. When multiple antennas were employed, both a time- and spatial-domain uncertainty region was considered. An entire angular uncertainty region may be divided into $L_{a}$ angular cells by focusing beams on different directions ${ }^{9}$, whilst the time-domain uncertainty region can be divided into $L_{t}$ delay cells covering the entire length of a spreading sequence [26]. Even though some of the papers in the literature have assumed a UL scenario, the proposed schemes are not strictly limited to the UL - they may also be tailored to MSs having multiple antennas. Finally, in [27], code acquisition schemes utilising both conventional singleantenna (1-D) and multielement antenna (2-D) aided schemes have been considered in the context of the UL. A datadirected-code acquisition approach was advocated, where the current users' detected information is exploited to enhance the quality of the received signal for the sake of detecting new users or paths. More explicitly, the reliably detected signals were subtracted from the composite received signal. Naturally, the employment of this technique is only feasible, if the UL receiver of the BS is capable of invoking multiuser detection.

\section{B. Code Acquisition in Co-located MIMOs}

Before describing the scenarios to be considered, our assumptions are stipulated as follows. The total allocated power is equally shared by the $P$ transmit antennas. The spacing of the multiple transmit antennas at the BS and the multiple receive antennas at the MS are assumed to be $10 \lambda$ and $0.5 \lambda$, respectively. It is also assumed that the BS antennas have a high elevation. The tapped-delay line channel model generates $L$ multi-path signals arriving with a time delay of

\footnotetext{
${ }^{9}$ The number of angular uncertainty regions may be determined by using a direction of arrival aided estimator or by a straightforward division of the entire angular uncertainty region into smaller cells based on classic fixed-beam aided approaches.
} 


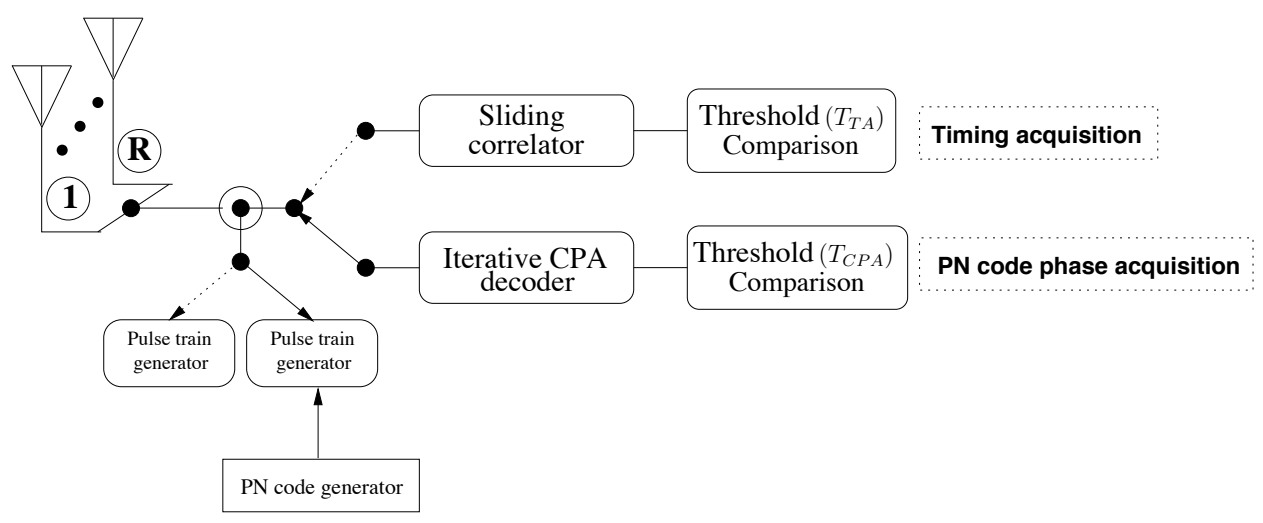

Fig. 17. Schematic of a receiver proposed for a SIMO scenario.

TABLE IV

Comparisons among the Mean Acquisition Time (MAT), Memory Complexity (MC) and Computational Complexity (CC) in TERMS OF SERIAL, HYBRID, PARALLEL AND ITERATIVE MP ACQUISITOIN SCHEMES

\begin{tabular}{|l|l|l|l|l|}
\hline & Serial & Hybrid $\left(H_{p}=8\right)$ & Parallel & Iterative MP \\
\hline MAT & $2^{r-1} \cdot N T_{c}$ & $\left(2^{r-1} \cdot N T_{c}\right) / H_{p}$ & $N T_{c}$ & $N T_{c}$ \\
& $\left(4.19 * 10^{6} T_{c}\right)$ & $\left(5.24 * 10^{5} T_{c}\right)$ & $\left(256 \cdot T_{c}\right)$ & $\left(256 \cdot T_{c}\right)$ \\
\hline MC & 1 & $H_{p}(8)$ & $2^{r}(32768)$ & $7 \cdot N(1792)$ \\
\hline CC & $2^{r-1} \cdot N$ & $2^{r-1} \cdot N$ & $2^{r} \cdot N$ & $1700 \cdot N$ \\
& $\left(4.19 * 10^{6}\right)$ & $\left(4.19 * 10^{6}\right)$ & $\left(8.39 * 10^{6}\right)$ & $\left(4.35 * 10^{5}\right)$ \\
\hline
\end{tabular}

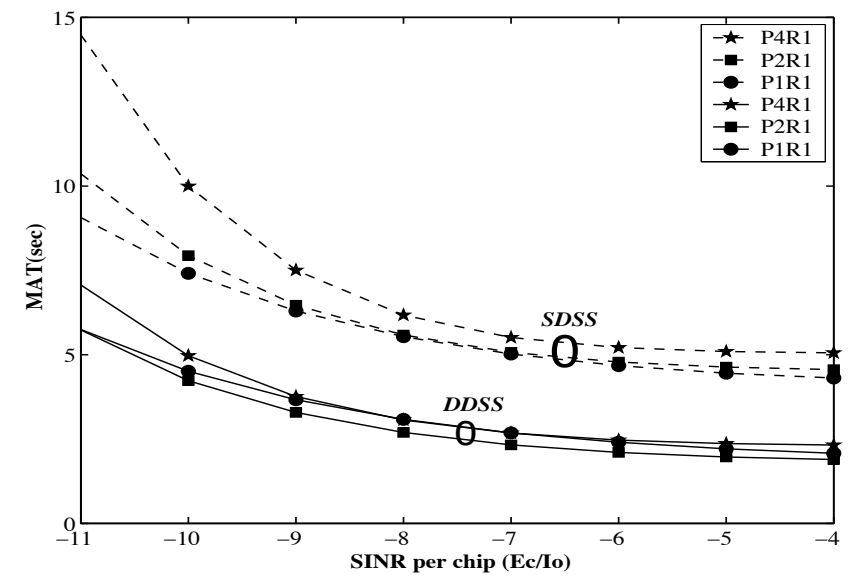

Fig. 18. MAT versus $E_{c} / I_{0}$ performance comparison between the NC-based DDSS and SDSS code acquisition schemes parameterised with the number of transmit antennas.

$\tau_{l}$ [16], [22], where $L$ is the number of multi-path signals received. In order to highlight our investigations of both the co-located and cooperative NC MIMO aided schemes, the characteristics of the co-located and cooperative NC MIMO aided code acquisition schemes is emphasised in terms of the achievable MAT performance in Figures 18 and 21, respectively.

Figure 18 illustrates the attainable MAT versus $E_{c} / I_{0}$ performance of the NC-based DDSS and SDSS code acquisition schemes as a function of the number of transmit antennas for $P=1,2$ and 4 as well as that of the number of receive antennas for $R=1$. In the results of Figure 18, the solid lines indicate the performance curves of the DDSS schemes, whereas the dashed lines represent the performance curves of the SDSS schemes. Observe in Figure 18 that as the number of transmit antennas is decreased, despite the potentially reduced transmit diversity gain, we experience an improved MAT performance for the single-path scenario of the SDSS scheme. Similarly to the conclusions of the SDSS scenario, as the number of transmit antennas is decreased, all the curves seen in Figure 18 for the single-path scenario of the DDSS scheme illustrate an improved MAT performance. However, a useful transmit diversity gain is experienced only for the case of ' $P 2 R 1^{\prime}$, and even this gain was limited to the specific SINR range of -4 and $-11 \mathrm{~dB}$. To illustrate the above fact a little further, in the case of ' $P 2 R 1^{\prime}$ the DDSS scheme exhibits a better MAT performance in comparison to the ' $P 1 R 1^{\prime}$ arrangement across the specific SINR range shown in Figure 18. It is worth noting that the total uncertainty region of this scenario was assumed to entail 65,534 hypotheses for Figure 18. This fact clearly implies that DDSS benefits from a significantly higher diversity gain than SDSS. The performance degradation imposed by employing multiple antennas becomes more drastic, as the number of transmit antennas is increased for both the SDSS and DDSS schemes, since the length of coherent summation is limited by the clock-drift-induced frequency mismatch. Furthermore, the associated MAT performance discrepancy between the SDSS and DDSS schemes becomes more drastic.

The main reasons for the above-mentioned performance trends may be further justified by information theoretic considerations in the NC MIMO aided scenarios considered [82]-[84]. The characteristics of the NC MIMO aided scenarios may be summarised as follows:

Wireless systems employing MIMO exhibit a high capacity, provided that the channel is known to the receiver [85]. By contrast, a NC MIMO aided scheme, which does not rely on any channel knowledge has a lower capacity [82]-[84]. However, it was argued in [83] that there is no reason for using more than $T_{\text {sym }} / 2$ number of transmit antennas, where 
$T_{\text {sym }}=2$ was the specific number of symbols over which integration was carried out, because the number of degrees of freedom increases with $T_{\text {sym }} / 2$, but only until the number of transmit antennas $P$ approaches $T_{\text {sym }} / 2$ [83]. Furthermore, at low SINRs the mutual information between the transmitter and receiver is maximised by using a single transmit antenna, because the mutual information bounds were shown to be decreasing functions of $P$ [84]. This implies that using multiple transmit antennas provides no MAT performance gain in the low SINR region, in fact, rather surprisingly, it leads to an MAT performance degradation. Finally, in the medium SINR range a maximum of $T_{\text {sym }}$ transmit antennas is worth employing for the sake of achieving an MAT performance gain, because the capacity achieved for $P>T_{\text {sym }}$ is the same as that achieved for $P=T_{\text {sym }}$ [82]. This indicates that $P=2$ transmit antennas are capable of achieving an improved MAT performance in the SINR region of -4 and -11 $\mathrm{dB}$, as demonstrated for the single-path scenario of Figure 18.

\section{Code Acquisition in Cooperative MIMOs}

In wireless networks, fading constitutes one of the main sources of channel-induced impairments. A powerful technique of overcoming the fading imposed by multi-path propagation is constituted by spatial diversity invoking MIMO, which has attracted substantial research interests [86]-[88]. Furthermore, the substantial appeal of MIMOs is that their capacity increases linearly with the SINR, as opposed to the more modest logarithmic increment of the classic ShannonHartley law, which may be readily elucidated by assigning the increased transmit power to an additional antenna and therefore linearly increasing the throughput [89]. However, in realistic propagation environments, the multiple antenna's signals typically become correlated owing to the sizelimitation of the MS and BS. Hence the spatial diversity gain of independently faded signals is often eroded. This phenomenon is typically imposed by shadow fading. In order to cope with this problem, various cooperative and Relay Station (RS)-aided transmission schemes have been proposed [90]-[92]. In lowcomplexity cooperative systems a MS receives the two-hop DL signal via the RS as well as the directly detected DL signal of the BS. Since these two signals generally arrive through completely different -rather than correlated- propagation paths, cooperative transmission becomes capable of mitigating the above-mentioned correlated shadow fading effects. Furthermore, exploiting the intermediate RS, cooperative transmission has the potential of extending the cell area and/or of improving the quality of cell-edge coverage, which results in requiring a reduced number of BSs. The RS in cooperative systems filters the signal received from the BS and retransmits it to the MS. The relaying schemes are commonly classified into two types: Amplify-and-Forward (AF) as well as Decodeand-Forward (DF) regimes [91], [92]. In the AF scheme, the RS simply retransmits the scaled version of the encountered signal and hence the noise component may also be amplified. By contrast, the DF aided RS fully decodes the received signal and forwards the re-encoded version in order to avoid the noise amplification. However, the DF strategy undoubtedly increases the complexity of RSs. In cooperative or distributed MIMO

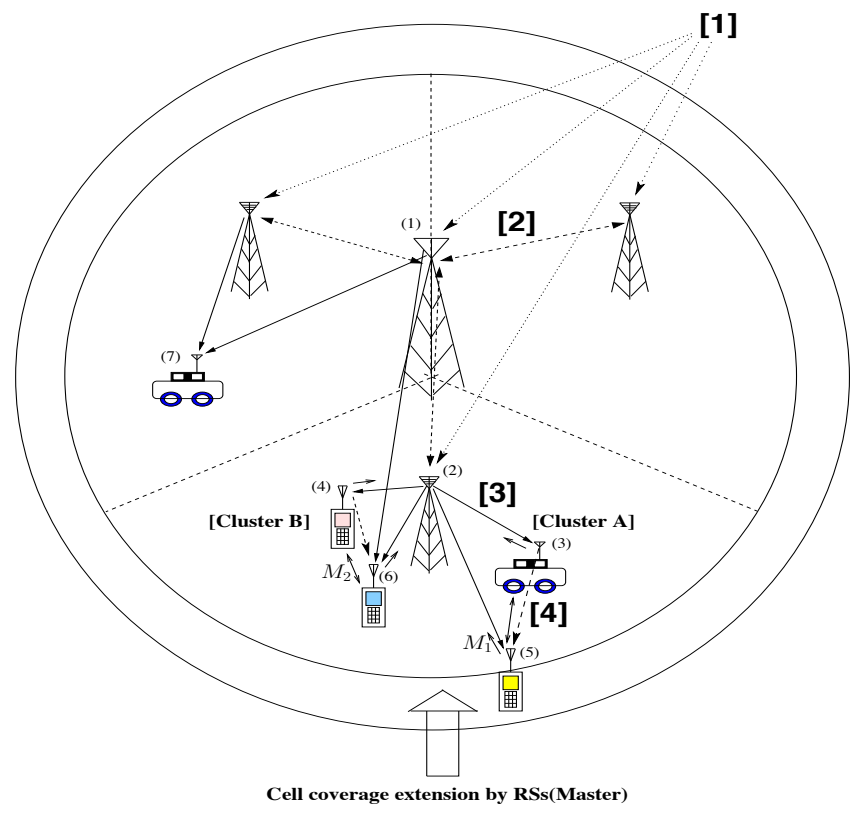

Fig. 19. Code acquisition scenario in cooperative MIMO environments, which constitutes three stages when considering a couple of RSs.

aided scenarios, the RS may be constituted by an intermediate MS that is currently not engaged in active communication or by a fixed RS that is installed at a specific position in a cell. However, for the sake of creating high-reliability RSs, we consider fixed RSs as the master of a specific RS-aided network [92]. It is also assumed that there is a LOS path between the master RSs as well as the BS [92].

Our detailed scenario is demonstrated in Figure 19. Figure 19 illustrates our three-stage code acquisition scenario encountered in fixed RS-aided environments, when considering several RSs located in a cell, where (1) represents a BS, (2) indicates a RS being the master of the RS-aided network, (3) is a slave RS of the RS-aided network, (4) represents another slave RS of the RS-aided network, (5) indicates a MS travelling at low speed, (6) is another MS roaming at low speed and (7) represents a MS moving at high speed. The traffic cell is divided into three sectors and a fixed $\mathrm{RS}$ is employed in each sector. We assume that both the BS and the RS have a single transmit antenna. It is also assumed that the BS is capable of supporting all the functions required. Furthermore, both the BS and master RSs are capable of supporting handover functionalities, whilst the master RS supports both AF, DF as well as pilot transmission. We also assume that some of the slave RSs support AF, DF as well as signalling message transmission for the RS-aided cluster, while other slave RSs may only support AF and signalling message transmission. Only MSs travelling at low speed can be included in a cluster supported by slave RSs. It is worth noting that a substantial cell-size extension may be achieved by fixed master RSs. Furthermore, both the master and slave RSs are capable of achieving an improved throughput and a cooperative diversity gain.

In Figure 19 the bold mark [1] suggests that global positioning system aided synchronisation is employed for both the BS and fixed master RSs, the bold mark [2] indicates the 
required initial control procedures to be carried out between the BS and fixed RSs, whilst the bold mark [3] portrays the procedures associated with a specific master and the slaves in a RS-aided temporary cluster. Finally, the bold mark [4] illustrates the communication procedures employed by the RSs and a specific MS. The bottom sector of Figure 19 depicts two clusters represented as Cluster A and B, respectively. Cluster A exemplifies a scenario, where only pilot transmission from a master RS is used for code acquisition in support of a MS located at the extended cell edge, while cluster B represents a scenario, where the BS transmits pilots in order to support the code acquisition and the master RSs also contribute towards supporting the code acquisition procedure. Within each cluster, the small dashed arrow indicates that only control information associated with a specific RS-aided cluster is sent without employing pilot transmission. Furthermore, the arrow $M_{1}$ of Figure 19 represents the signalling message, which is a specific PN code based preamble and provides MS identifier information in the UL in order to register both the slaves and the MSs. Finally, the arrow $M_{2}$ of Figure 19 represents another signalling message, which is another specific PN code based preamble and provides MS identifier information in a specific RS-aided cluster for the sake of acquiring timing information between the slaves and the MSs. The top left sector of Figure 19 depicts a scenario, when considering a MS roaming at high speed, in which the employment of fixed RSs is only possible, because the high speed of the MS does not allow a RS-aided cluster to be established. To illustrate this scenario a little further, a PN sequence having the same code phase offset is used by both the BS and the fixed master RSs in order to acquire GPS aided synchronisation. Different Walsh codes are assigned to both the BS and the fixed master RSs, respectively, where the BS and master RSs are fixed at a specific position.

Based on the above elaborations, our three-stage code acquisition scheme designed for RS-aided inter-cell synchronous DS-CDMA DL systems is summarised as follows:

1) First stage: Timing acquisition between the BS and master RSs $\Rightarrow$ Both are capable of extracting timing information from the received signal because they are fixed and it is already known which one was assigned a specific Walsh code for its future communications.

2) Second stage: Timing acquisition between the BS and master RSs as well as MSs. $\Rightarrow$ In this scenario, the MSs are capable of benefitting from cooperative diversity. We will analyse this particular scenario in detail.

3) Third stage: Timing acquisition among the RSs (master and slave) and MSs. $\Rightarrow$ By employing the $M_{1}$ message of Figure 19 in the UL, the slave RSs and the MSs are registered with a specific master RS. When using the $M_{2}$ message of Figure 19 among the slave RSs and the MSs, timing acquisition is accomplished within a previously established cluster. A new broadcast downlink channel is used for distributing the cluster assignment or grouping information. More specifically, the grouping information includes assignment of slave RSs and unique identification information for each element of the cluster. Based on the information provided by the broadcast channel, the $M_{2}$ messages

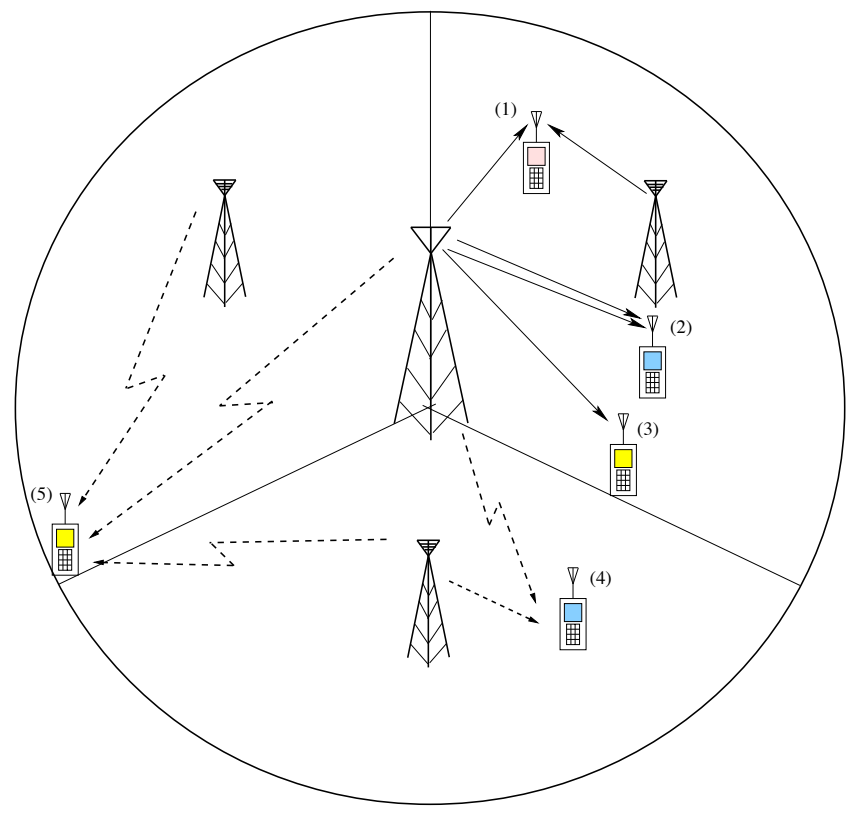

Fig. 20. Code acquisition scenarios in cooperative MIMO environments, which encompasses three normalised-power scenarios (denoted as (1), (2) and (3)) and two increased-power ones (denoted as (4) and (5)) when considering one or two RSs.

of Figure 19 are exchanged in order to establish timing acquisition among the constituents of a specific cluster.

Our discussions here are essentially confined to the second stage designed for employment in an RS-aided code acquisition scheme.

Figure 20 illustrates a number of code acquisition scenarios encountered in fixed RS-aided environments, when considering one or two RSs. The traffic cell is divided into three sectors and a fixed RS is employed in each sector. We assume that both the BS and the RS have a single transmit antenna, except for a co-located scenario having two transmit antennas. The top right sector of Figure 20 depicts three scenarios, where the total allocated power is equally shared by the transmit antennas in both the co-located and cooperative transmission scenarios (termed as 'normalisedpower scenario') as follows:

- First scenario (denoted as (1) in Figure 20) : The MS receives the DL signals from both the RS located at the same sector's centre as well as from the BS and processes them in order to attain reliable code acquisition.

- Second scenario (denoted as (2) in Figure 20) : A co-located scenario having two transmit antennas is considered.

- Third scenario (denoted as (3) in Figure 20) : A SISO scenario is used as a benchmarker.

By contrast, in both the fourth and fifth scenarios it is assumed that the total allocated power is also proportionately increased according to the number of RSs (referred to as 'increased-power scenario'), as detailed below:

- Fourth scenario (denoted as (4) in Figure 20) : It is exactly the same as the first one, except for its different power allocation.

- Fifth scenario (denoted as (5) in Figure 20) : When the MS is located at the edge of the top left sector 


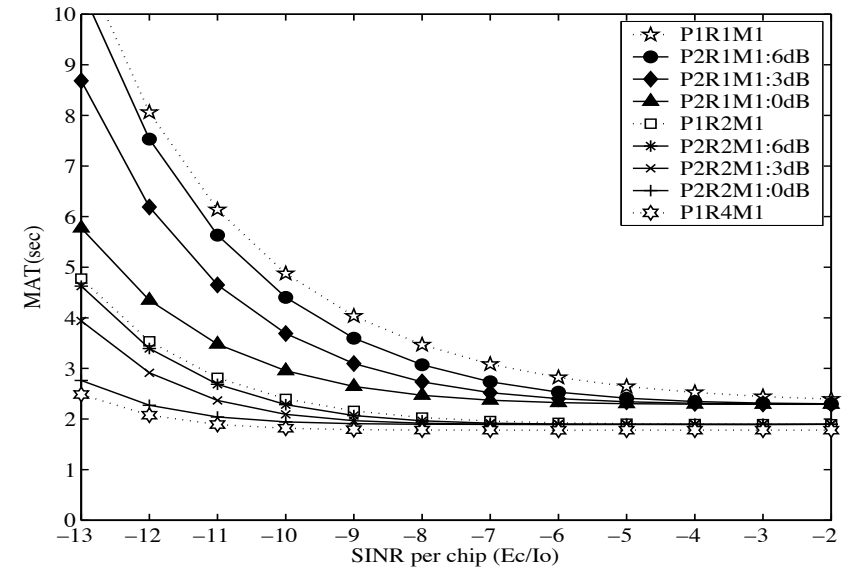

Fig. 21. MAT versus SINR per chip performance of the code acquisition system for DDSS parameterised with link imbalance and the number of receive antennas for one RS and a single path (increased-power scenario).

of Figure 20, the signal received from the RS at the adjacent sector's centre seen at the bottom of Figure 20 also arrives at the MS with a substantial signal strength.

Furthermore, in the fifth case, this particular MS has to combine the signals received from all the three transmitters for the sake of reliable code acquisition. The average strength of the RS' signal varies depending on the location of the MS. Explicitly, when the MS is located near the cell-edge, the signal received from the RS generally has a higher signal strength with respect to that of the BS, which results in the phenomenon often referred to as link imbalance between the signals received by the MS.

Figure 21 illustrates the achievable MAT versus SINR per chip performance of the DDSS code acquisition scheme parameterised with both the grade of link imbalance and with the number of receive antennas for a single RS and a single propagation path, when considering the increasedpower scenario. Observe in Figure 21 that when the link imbalance is decreased, we experience an improved MAT performance. When there is no link imbalance, the MAT performance approaches that of having two receive antennas. On the other hand, in case of having a $6 \mathrm{~dB}$ imbalance, only a marginal diversity gain is achieved, hence the attainable MAT performance improvement also becomes negligible. The above-mentioned conclusions explicitly demonstrate that employing a single $\mathrm{RS}$ is beneficial in terms of the achievable MAT performance, and as expected, the achievable improvements depend on the value of the link imbalance. However, employing a single RS cannot guarantee maintaining a high diversity gain due to the fluctuation of the RS's link quality.

\section{Characteristics of Code Acquisition in Both Co-located and Cooperative MIMOs}

Ironically, based on [28]-[31], [33] and Figure 20, our findings suggest that increasing the number of transmit antennas in a co-located MIMO-aided SC-DS-CDMA system results in combining the low-energy, noise-contaminated signals of the transmit antennas. Furthermore, increasing both the number of transmit antennas and that of the subcarriers in a co-located MIMO-aided MC-DS-CDMA system also results in combining the low-energy, noise-contaminated signals of both the transmit antennas and the subcarriers [28], [29]. This fact ultimately reduces the correct detection probability, and accordingly increases the MAT by an order of magnitude, when the SINR is relatively low. However, it is extremely undesirable to degrade the achievable acquisition performance, when the system is capable of attaining its target BER performance at reduced SINR values. This phenomenon also has a detrimental effect on the performance of Rake receiver based synchronisation, when the perfectly synchronised system is capable of attaining its target bit error rate performance at reduced SINR values, as a benefit of employing multiple transmit antennas and/or frequency diversity. Hence it may be concluded that the achievable cell coverage determined by the received pilot channel power may be reduced, as the number of transmit antennas is increased, which is a highly undesirable phenomenon, since it has grave repercussions in terms of having to tolerate a high number of handovers per cell.

In contrast to the detrimental effects of sharing the total transmit power across multiple transmit antennas in the colocated and cooperative MIMO based scenarios characterised in Figure 21 and [32], our findings suggest that employing distributed MIMO elements acting as RSs combined with multiple receive antennas leads to an improved MAT performance. However, these gains are only achievable, if the RSs can afford to contribute toward supplying the extra power used in the 'increased-power-scenario'. By contrast, when having a high link imbalance, only marginal MAT performance gains may be achieved, regardless whether single-path or multi-path propagation scenarios are considered. Therefore, in order to efficiently exploit the diversity benefits of RS-aided transmissions, the employment of at least two RSs might be recommended. When additionally invoking multiple colocated receive antennas at the MS, further diversity gains may be achieved.

Finally, when considering the design of co-located MIMO aided code acquisition schemes, the following guidelines may be inferred:

1) In general, coherently detected space-time transmission schemes benefit from having explicit knowledge of the channel impulse response. However, this is unavailable during the code-acquisition phase.

2) Using multiple transmit antennas typically leads to an MAT performance degradation, owing to the reduced per-antenna power, except for specific scenarios, when encountering a single-path environment, also depending on the allocated bandwidth. In the multi-path scenarios considered all the schemes fail to show a transmit diversity gain. Therefore, during code acquisition activating only a single transmit antenna might be recommended for the sake of maximising the achievable MAT performance of the code acquisition scheme investigated.

3) Using a relatively low number of chips, over which integration or accumulation is carried out imposes further limits on the attainable benefits of MIMO aided schemes [82], 
[83].

4) Employing the more sophisticated DDSS and/or DC schemes may provide a rather limited further diversity gain in comparison to using SDSS and/or NC schemes.

5) Exploiting multiple receive antennas increases the achievable receiver diversity gain and has the potential of compensating for the MAT degradation imposed by the low per-branch power of both multiple transmitters and multiple subcarriers.

6) Since no channel coding is used for the pilot signal, no time diversity gain associated with interleaving and channel coding can be achieved [93]. However, the MIMO aided code-acquisition schemes are only capable of achieving a rather limited time diversity, namely, using a sufficiently high number of PDI stages is also available for minimising MAT [3], [74].

7) When the detection threshold $\theta_{2}$ of Figure 3 is reduced, the resultant code phase estimate often cannot be confirmed by the verification stage of Figure 3 and hence the false alarm probability is increased. At the same time, the correct detection probability is also increased. However, when aiming for the best achievable MAT performance, the detection threshold optimisation has to strike a balance between increasing the false alarm probability and the correct detection probability, because after a false alarm event the system may require 1000 chip-durations to return to its search mode.

8) The effect of using a pair of fixed thresholds of $\theta_{1}$ and $\theta_{2}$ in Figure 3, which are optimised for a specific $E_{c} / I_{0}$ value also limits the attainable MAT performance, since the acquisition threshold should be optimised and controlled as a function of the $E_{c} / I_{0}$ value encountered.

9) For the sake of acquiring the exact timing information of the received paths without any potential performance degradation that might be imposed on the NC MIMO aided scenarios, specifically designed preambles, such as that of the primary-synchronisation channel of W-CDMA [39] combined with time switched transmit diversity [94] might be recommended, which is capable of achieving a diversity gain with the aid of a single transmit antenna [83], [84]. In practical scenarios, the received path timing differences of the signals arriving from multiple transmit antennas might be distributed within a fraction of a chip duration [95], although they may vary owing to the time-variant propagation delay, hence using multiple transmit antennas may degrade the performance further. In addition to initial acquisition, the classic pilot channel may also be used for other purposes, such as frequency error correction and channel estimation so as to support coherent MIMO aided scenarios [96].

Furthermore, the following conclusions may be inferred for the cooperative MIMO scenarios:

1) Sharing the total transmit power across multiple transmit antennas in the co-located and cooperative MIMO based scenarios leads to similarly detrimental effects.

2) Employing multiple relay stations increases the achievable transmitter diversity gain, as suggested by all the figures associated with the MAT performance.

3) The value of link imbalance gravely affects the achievable MAT performance. When having a high link imbalance, only marginal MAT performance gains may be achieved, regardless whether single-path or multi-path propagation scenarios are considered. Therefore, in order to efficiently exploit the diversity benefits of RS-aided transmissions, the employment of at least two RSs might be recommended.

4) When additionally invoking multiple co-located receive antennas at the MS, further diversity gains may be achieved.

\section{CONCLUSIONS}

In this article, we have provided a comprehensive survey of the associated open literature that is related to the principle of initial synchronisation in the single- and multi-carrier CDMA as well as DS-UWB DL. We have commenced our discourse by outlining the related basic terminology and definitions in Section II. We have limited our elaborations to the basic principles of code acquisition procedures, the interaction between the synchronisation module and Rake receiver, the associated decision strategy and the concept of MAT. Following these preliminaries, in Section III we proceeded to provide various search strategies, widely used detector structures, the initial and post-initial code acquisition as well as code acquisition schemes designed for both the MC-DS-CDMA DL and for DS-UWB DL. More specifically, we focused our attention on the literature concerning various search strategies. The underlying serial search based code acquisition was presented, followed by the parallel search (ML) based code acquisition scheme. Then the set of known sequential estimation based code acquisition schemes were classified into four categories. Finally, random search based code acquisition was presented. Then, we outlined a variety of widely used detector structures. Subsequently, we detailed NC code acquisition as well as both a chip based-DC detection scheme and a FPC based DC detection scheme. We also discussed the widely-used PDI concept of practical code acquisition receivers. Additionally, we summarised the historic evolution of sequential estimation based code acquisition as well as that of DC acquisition schemes in Tables II and III, respectively. We have provided a concise portrayal of both initial and post-initial code acquisition schemes designed for both the MC-DS-CDMA DL and for DS-UWB DL. More specifically, the last subsection constitutes an overview of a variety of schemes, such as iterative MP aided code acquisition and two-stage iterative arrangements. The subsection has portrayed how the most popular iterative detection schemes usually employed for channel coding schemes in the literature have been adopted in terms of code acquisition approaches [37], [49], [56], [76], [77], [79]-[81].

In Section IV, we have focused our attention on current research topics related to code acquisition in both the colocated and cooperative MIMOs. We proceeded by explaining some basic scecnarios regarding code acquisition in colocated MIMOs. More explicitly, we attempted to illustrate the behaviours of the NC co-located MIMOs in terms of code acquisition, then the related information theoretic considerations in the NC MIMO aided scenarios were portrayed in a concise manner. Finally, we have outlined a three-stage code acquisition scenario encountered in fixed RS-aided environments, closing with the characteristics of code acquisition in both the co-located and cooperative MIMOs. Based on our elaborations regarding the diverse code 
acquisition scenarios relying on both cooperative MIMOs and iterative acquisition schemes, a range of further sophisticated approaches may be considered for future research.

\section{GLOSSARY}

AF : Amplify-and-Forward

AFC : Automatic Frequency Control

BF : BeamForming

BS : Base Station

CDMA : Code Division Multiple Access

CFAR : Constant False-Alarm Rate

CN : Check Node

CPA : Code Phase Acquisition

DC : Differentially Coherent

DC-MF : Differential Correlations based Matched Filter

DC-MUSIC : Differential Correlations based MUltiple SIgnal Classification

DDSS : Double Dwell Serial Search

DF : Decode-and-Forward

DL : DownLink

DRSSE : Differential Recursive Soft Sequential Estimation

DSR : Decoder Shift Register

DS-UWB : Direct Sequence-Ultra WideBand

EGC : Equal Gain Combining

FPC : Full-Period Correlation

GP : Generator Polynomial

LDPC : Low-Density Parity-Check

LFSR : Linear Feedback Shift Register

LLR : Log-Likelihood Ratio

MAT : Mean Acquisition Time

MC-DS-CDMA : Multi Carrier-Direct Sequence-Code Division Multiple Access

MF : Matched-Filter

MIMO : Multiple-Input Multiple-Output

ML : Maximum Likelihood

MLD : Majority Logic Decoding

MLSR : Maximum Length (linear) Shift Register

MP : Message Passing

MRC : Maximum Ratio Combining

MS : Mobile Station

NC : Non-Coherent

P-C : Parity-Check

PCM : Parity Check Matrix

PDI : Post-Detection Integration

PN : Pseudo-Noise

PP : Primitive Polynomial

PPC : Partial-Period Correlation

RARASE : Recursion-Aided RASE

RASE : Rapid Acquisition by Sequential Estimation

RS : Relay Station

RSSE : Recursive Soft Sequential Estimation

SCDU : Soft-Chip Delay Unit

SDSS : Single Dwell Serial Search

SeC : Selection Combining

SIMO : Single-Input Multiple-Output

SINR : Signal-to-Interference plus Noise Ratio

SISO : Single-Input Single-Output

SI/SO : Soft-In/Soft-Out
SP : Serial-to-Parallel

TA : Timing Acquisition

UL : UpLink

VL : Variable Node

\section{REFERENCES}

[1] V. K. Garg, "IS-95 CDMA and cdma 2000: Cellular/PCS Systems Implementation", Prentice Hall PTR, 1999.

[2] J. S. Blogh and L. Hanzo, "Third Generation Systems and Intelligent Wireless Networking: Smart Antennas and Adaptive Modulation", John Wiley and Sons, 2002.

[3] A. J. Viterbi, "CDMA: Principles of Spread Spectrum Communication, Chapter 2 and 3", Addison-Wesley, 1995.

[4] R. L. Peterson and R. E. Ziemer and D. E. Borth, "Introduction to Spread Spectrum Communications", Prentice Hall Inc., 1995.

[5] M. K. Simon and J. K. Omura and R. A. Scholtz and B. K. Levitt, "Spread Spectrum Communications Handbook", McGraw-Hill Professional, 2001

[6] L. Hanzo and L. L. Yang and E.L. Kuan and K. Yen, "Single- and Multi- Carrier DS-CDMA", John Wiley \& Sons, 2003.

[7] S. Glisic, M.D. Katz, "Modeling of the Code Acquisition Process for Rake Receivers in CDMA Wireless Networks with Multipath and Transmitter Diversity", IEEE J. Sel. Areas Commun., vol. 19, no.1, Issue 1, 2001, pp21-32.

[8] S.H. Won and Y.J. Kim, "Performance Analysis of Multi-path Searcher for Mobile Station in W-CDMA System Employing Transmit Diversity", Electronics Letters, vol. 39, Issue 1, 2003, pp137-139.

[9] A. Polydoros and S. Glisic, "Code Synchronization: a Review of Principles and Techniques", IEEE Proceedings of the International Symposium on Spread Spectrum Techniques and Applications (ISSSTA), 4-6 July, 1994, pp115-137

[10] E. A. Sourour and S. C. Gupta, "Direct-Sequence Spread-Spectrum Parallel Acquisition in a Fading Mobile Channel", IEEE Trans. Commun., vol. 38, no.7, 1990, pp992-998.

[11] K. K. Chawla and D. V. Sarwate, "Parallel Acquisition of PN Sequences in DS/SS Systems", IEEE Trans. Commun., vol. 42, no.5, 1994, pp2155-2164.

[12] R. B. Ward, "Acquisition of Pseudonoise Signals by Sequential Estimation", IEEE Trans. Commun. Technol., vol. 13, no.4, 1965, pp475-483.

[13] R. Ward and K. Yiu, "Acquisition of Pseudonoise Signals by Recursion-Aided Sequential Estimation”, IEEE Trans. Commun., vol. 25, no.8, 1977, pp784-794.

[14] L-L Yang, L. Hanzo, "Acquisition of m-sequences using recursive soft sequential estimation", IEEE Trans. Commun., vol. 52, no.2, 2004, pp199-204.

[15] H. R. Park and B. J. Kang, "On Serial Search Code Acquisition for Direct-Sequence Spread Spectrum System: An Application to IS-95 CDMA system", IIEEE Vehicular Technology Conference (VTC), 25-28 July, 1995, pp291-295.

[16] H.R. Park, "Performance Analysis of a Double-Dwell Serial Search Technique for Cellular CDMA Networks in the Case of Multiple Pilot Signals", IEEE Trans. Veh. Technol., vol. 48, no.6, Issue 6, 1999, pp1819-1830.

[17] C. D. Chung, "Differentially Coherent Detection Technique for Direct-Sequence Code Acquisition in a Rayleigh Fading Mobile Channel", IEEE Trans. Commun., vol. 43, no.234, 1995, pp11161126

[18] M. H. Zarrabizadeh and E. S. Sousa, "A Differentially Coherent PN Code Acquisition Receiver for CDMA Systems", IEEE Trans. Commun., vol. 45, no.11, 1997, pp1456-1465.

[19] J. C. Lin, "Differentially Coherent PN Code Acquisition with FullPeriod Correlation in Chip-Synchronous DS/SS Receivers", IEEE Trans. Commun., vol. 50, no.5, 2002, pp698-702.

[20] L. L. Yang and L. Hanzo, "Serial Acquisition Performance of Single-Carrier and Multicarrier DS-CDMA over Nakagami-m Fading Channels", IEEE Trans. Wireless Commun., vol. 1, no.4, 2002, pp692-702.

[21] D. Lee and L. B. Milstein, "Analysis of a Multicarrier DS-CDMA Code Acquisition System", IEEE Trans. Commun., vol. 47, no.8, 1999, pp1233-1244.

[22] L. L. Yang and L. Hanzo, "Serial Acquisition of DS-CDMA Signals in Multipath Fading Mobile Channels", IEEE Trans. Veh. Technol., vol. 50, no.2, 2001, pp617-628. 
[23] D. Dlugos and R. Scholtz, "Acquisition of Spread Spectrum Signals by An Adaptive Array", IEEE Trans. Acoust., Speech Signal Process., vol. 37, no.8, 1989, pp1253-1270.

[24] M. Katz, J. Iinatti and S. Glisic, "Two-Dimensional Code Acquisition in Time and Angular Domains", IEEE J. Sel. Areas Commun., vol. 19, no.12, 2001, pp2441-2451.

[25] B. Wang and H. Kwon, "PN Code Acquisition for DS-CDMA Systems Employing Smart Antennas-Part II", IEEE J. Wireless Commun., vol. 2, no.1, 2003, pp108-117.

[26] H. Puska, J. Iinatti and H. Saarnisaari, "Serial Search and Maximum Selection Based Code Acquisition Techniques for Single and Multi Antenna Systems", IEEE J. Wireless Commun., vol. 8, no.5, 2009, pp2317-2327.

[27] M. Reed, L. Hanlen and G. Corazza, "Return-Link Code Acquisition for 1-D and 2-D With DS-CDMA for High-Capacity Multiuser Systems", IEEE Trans. Veh. Technol., vol.57, no.1, 2008, pp324334.

[28] S.H. Won and L. Hanzo, "Differentially Coherent Code Acquisition in the MIMO-Aided Multi-Carrier DS-CDMA Downlink", IET Communications, vol. 1, no 4, August 2007, pp662-670.

[29] S.H. Won and L. Hanzo, "Non-Coherent Code Acquisition in the Multiple Transmit/Multiple Receive Antenna Aided Single- and MultiCarrier DS-CDMA Downlink", IEEE Trans. Wireless Commun., vol. 6, no 11, November 2007, pp3864-3869.

[30] S.H. Won and L. Hanzo, "Analysis of Serial-Search-Based Code Acquisition in the Multiple-Transmit/Multiple-Receive-Antenna-Aided DS-CDMA Downlink", IEEE Trans. Veh. Technol., vol. 57, no. 2, 2008, pp1032-1039.

[31] S.H. Won and L. Hanzo, "Non-Coherent and Differentially Coherent Code Acquisition in MIMO Assisted DS-CDMA Multi-Path Downlink Scenarios", IEEE Trans. Wireless Commun., vol. 7, no.5, part 1, 2008, pp1585-1593.

[32] S.H. Won, K.C. Lee and L. Hanzo, "Initial Code Acquisition in the Cooperative Non-coherent MIMO DS-CDMA Downlink", IEEE Trans. Veh. Technol., vol. 58, no.3, March 2009, pp1387-1395.

[33] S.H. Won and L. Hanzo, "Initial and Post-Initial Code Acquisition in the Non-Coherent Multiple Input/Multiple Output Aided DS-CDMA Downlink", IEEE Trans. Veh. Technol., vol. 58, no 5, June 2009, pp $2322-2330$

[34] S. Roy, J.R. Foerster, V.S. Somayazulu and D.G. Leeper, "Ultrawideband Radio Design: The Promise of High-Speed, ShortRange Wireless Connectivity", Proc. IEEE, vol. 92, no.2, 2004, .pp295-311.

[35] R.C. Qiu, H. Liu and X. Shen, "Ultra-Wideband for Multiple Access Communications", IEEE Commun. Mag., vol. 43, no.2, 2005, pp80-87.

[36] S.R. Aedudodla, S. Vijayakumaran and T.F. Wong, "Timing Acquisition in Ultra-Wideband Communication Systems", IEEE Trans. Veh. Technol., vol. 54, no. 5, 2005, pp1570-1583.

[37] K.M. Chugg and M. Zhu, "A New Approach to Rapid PN Code Acquisition Using Iterative Message Passing Techniques", IEEE J. Sel. Areas Commun., vol. 23, no.5, 2005, pp884-897.

[38] G.E. Corazza and C. Caini and A. Vanelli-Coralli and A. Polydoros, "DS-CDMA Code Acquisition in the Presence of Correlated FadingPart I: Theoretical Aspects", IEEE Trans. Commun., vol. 52, no.7, 2004, pp1160-1168.

[39] Y.-P.E. Wang and T. Ottosson, "Cell Search in W-CDMA", IEEE J. Sel. Areas Commun., vol. 18, no.8, 2000, pp1470-1482.

[40] H. R. Park and B. J. Kang, "On the Performance of a MaximumLikelihood Code-Acquisition Technique for Preamble Search in a CDMA Reverse Link", IEEE Trans. Veh. Technol., vol. 47, no.1, 1998, pp65-74.

[41] R. N. McDonough and A. D. Whalen, "Detection of Signals in Noise, Chapter 5", Academic Press, 1995.

[42] C. Caini and G.E. Corazza and A. Vanelli-Coralli, "DS-CDMA Code Acquisition in the Presence of Correlated Fading-Part II: Application to Cellular Networks", IEEE Trans. Commun., vol. 52, no.8, 2004, pp1160-1168.

[43] W. Suwansantisuk and M. Win, "Multipath Aided Rapid Acquisition: Optimal Search Strategies", IIEEE Trans. Inf. Theory, vol. 53, no.1, 2007, pp174-193.

[44] C. Kilgus, "Pseudonoise Code Acquisition Using Majority Logic Decoding", IEEE Trans. Commun., vol. 21, no.6, 1973, pp772-774.

[45] C. Berrou and A. Glavieux and P. Thitimajshima, "Near-Shannon Limit Error-Correcting Coding and Ddecoding: Turbo-Codes. 1", Proceedings of the IEEE International Conference on Communications (ICC), 23-26 May, 1993, pp1064-1070.
[46] C. Berrou and A. Glavieux, "Near Optimum Error-Correcting Coding and Ddecoding: Turbo-Codes", IEEE Trans. Commun., vol. 44, no.10, 1996, pp1261-1271.

[47] B. Sklar, "A Primer on Turbo Code Concepts", IEEE Commun. Mag., vol. 35, no.12, 1997, pp94-102.

[48] S. Benedetto and D. Divsalar and G. Montorsi and F. Pollara, "A Soft-Input Soft-Output APP Module for Iterative Decoding of Concatenated Codes", IEEE Communications Letters, vol. 1, no.1, 1997, pp772-774.

[49] J.P. Woodard and L. Hanzo, "Comparative Study of Turbo Decoding Techniques: An Overview", IEEE Trans. Veh. Technol., vol. 49, no.6, 2000, pp2208-2233.

[50] L. L. Yang and L. Hanzo, "Iterative Soft Sequential Estimation Assisted Acquisition of m-Sequences", IEE Electronics Letters, vol. 38, no.24, 2002, pp1550-1551.

[51] L. L. Yang and L. Hanzo, "Differentially Acquisition of m-Sequences Using Recursive Soft Sequential Estimation", IEEE Trans. Wireless Commun., vol. 4, no.1, 2005, pp128-136.

[52] S. Lin and D. J. Costello, "Error Control Coding, 2nd ed.", Prentice Hall, 2004

[53] J. H. Lee and I. H. Song and S. R. Park and J. M. Lee, "Rapid Acquisition of PN Sequences with a New Decision Logic", IEEE Trans. Veh. Technol., vol. 53, no.1, 2004, pp49-60.

[54] H. Tanaka and K. Furusawa and S. Kaneku, "A Novel Approach to Soft Decision Decoding of Threshold Decodable Codes", IEEE Trans. Inf. Theory, vol. 26, no.2, 1980, pp244-246.

[55] J. Hagenauer and E. Offer and L. Papke, "Iterative Decoding of Binary Block and Convolutional Codes", IEEE Trans. Inf. Theory, vol. 42, no.2, 1996, pp429-445.

[56] O.W. Yeung and K.M. Chugg, "An Iterative Algorithm and Low Complexity Hardware Architecture for Fast Acquisition of Long PN Codes in UWB Systems", Springer J. VLSI and Signal Processing (Special Issue on UWB Systems), vol. 43, no.1, 2006, pp25-42; www.springerlink.com.

[57] A.F. Molisch and J.R. Foerster and M. Pendergrass, "Channel Models for Ultrawideband Personal Area Networks", IEEE Wireless Commun., vol. 10, no.6, 2003, pp14-21.

[58] I. Ramachandran and S. Roy, "On Acquisition of Wideband Directsequence Spread Spectrum Signals", IEEE Trans. Wireless Commun., vol. 5, no.6, 2006, pp1537-1546.

[59] L. S. Lee and J. H. Chiu, "An Improved Sequential Estimation Scheme for PN Acquisition", IEEE Trans. Commun., vol. 36, no.10, 1988, pp1182-1184.

[60] R. T. Barghouthi and G. L. Stüber, "Rapid Sequence Acquisition for DS/CDMA Systems Employing Kasami Sequences", IEEE Trans. Commun., vol. 42, no.234, 1994, pp1957-1968.

[61] H. S. Liaw and C. D. Chung, "Differentially Coherent Correlation Technique for DS-SS Code Acquisition in a Fast Rayleigh Fading Channel", IEE Electronics Letters, vol. 30, no.21, 1994, pp17381740.

[62] T. Ristaniemi and J. Joutsensalo, "Code Timing Acquisition for DS-CDMA in Fading Channels by Differential Correlations", IEEE Trans. Commun., vol. 49, no.5, 2001, pp899-910.

[63] Y. K. Jeong and O. S. Shin and K. B. Lee, "Fast Slot Synchronization for Intercell Asynchronous DS/CDMA Systems", IEEE Trans. Wireless Commun., vol. 1, no.2, 2002, pp353-360.

[64] J. C. Lin, "Differentially Coherent PN Code Acquisition with FullPeriod Correlation in Chip-Asynchronous DS/SS Receivers", IEEE Trans. Veh. Technol., vol. 51, no.6, 2002, pp1596-1599.

[65] O. S. Shin and K. B. Lee, "Differentially Coherent Combining for Double-Dwell Code Acquisition in DS-CDMA Systems", IEEE Trans. Commun., vol. 51, no.7, 2003, pp1046-1050.

[66] 3GPP, "3GPP TS 25.101 V4.11.0, User Equipment (UE) Radio Transmission and Reception (FDD)", 3GPP, 2006.

[67] V. M. DaSilva and E. S. Sousa, "Performance of Orthogonal CDMA Codes for Quasi-Synchronous Communication Systems", Proc. ICUPC, 12-15 October, 1993, pp995-999.

[68] S. Kondo and B. Milstein, "Performance of Multicarrier DS CDMA Systems", IEEE Trans. Commun., vol. 44, no.2, 1996, pp238-246.

[69] E. A. Sourour and M. Nakagawa, "Performance of Orthogonal Multicarrier CDMA in a Multipath Fading Channel", IEEE Trans. Commun., vol. 44, no.3, 1996, pp356-367.

[70] S. Hara and R. Prasad, "Overview of Multicarrier CDMA", IEEE Commun. Mag., vol. 35, no.12, 1997, pp126-133.

[71] J. Ibrahim and R. M. Buehrer, "Two-Stage Acquisition for UWB in Dense Multipath", IEEE J. Sel. Areas Commun., vol. 24, no.4, 2006, pp801-807. 
[72] J. Furukawa and Y. Sanada and T. Kuroda, "Novel Initial Acquisition Scheme for Impulse-based UWB Systems", Proc. 2004 Intl Workshop Ultra Wideband Systems, 18-21 May, 2004, pp278-282.

[73] S.R. Aedudodla and S. Vijayakumaran and T.F. Wong, "UltraWideband Signal Acquisition with Hybrid DS-TH Spreading", IEEE Trans. Wireless Commun., vol. 5, no.9, 2006, pp2504-2515.

[74] B. G. Lee and B. H. Kim, "Scrambling Techniques For CDMA Communications, Chapter 2 and 3", Kluwer Academic Publishers, 2001.

[75] J. S. Lee and L. E. Miller, "CDMA Systems Engineering Handbook, Chapter 11, CDMA Optimization Issues", Artech House Publishers, 1998

[76] S.H. Won and L. Hanzo, "Iterative Code Acquisition for the DSUWB Downlink Using Multiple-Component Decoders", Electronics Letters, vol. 44, no 2, January 2008, pp 162-163.

[77] S.H. Won and L. Hanzo, "Initial Acquisition Performance of the Multiple Receive Antenna Assisted DS-UWB Downlink Using Search Space Reduction and Iterative Code Phase Estimation", IEEE Trans. Wireless Commun., vol. 8, no 1, January 2009, pp 386-395.

[78] R. Tanner, "A Recursive Approach to Low Complexity Codes", IEEE Trans. Inf. Theory, vol. 27, no.5, 1981, pp533-547.

[79] F.R. Kschischang and B.J. Frey and H.-A. Loeliger, "Factor Graphs and the Sum-Product Algorithm", IEEE Trans. Inf. Theory, vol. 47, no.2, 2001, pp498-519.

[80] F.R. Kschischang and B.J. Frey, 'Iterative Decoding of Compound Codes by Probability Propagation in Graphical Models", IEEE J. Sel. Areas Commun., vol. 16, no.2, 1998, pp219-230.

[81] M. Schwartz and A. Vardy, "On the Stopping Distance and the Stopping Redundancy of Codes", IEEE Trans. Inf. Theory, vol. 52, no.3, 2006, pp922-932.

[82] T.L. Marzetta and B.M. Hochwald, "Capacity of a Mobile MultipleAntenna Communication Link in Rayleigh Flat Fading”, IEEE Trans. Inf. Theory, vol. 45, no.1, 1999, pp139-157.

[83] L. Zheng and D. N. C. Tse, "Communication on the Grassman Manifold: A Geometric Approach to the Noncoherent MultipleAntenna Channel", IEEE Trans. Inf. Theory, vol. 48, no.2, 2002, pp359-383.

[84] C. Rao and B. Hassibi, "Analysis of Multiple-Antenna Wireless Links at Low SNR", IEEE Trans. Inf. Theory, vol. 50, no.9, 2004, pp2123-2130.

[85] G. J. Foschini, "Layered Space-Time Architecture for Wireless Communication in a Fading Environment When Using MultiElement Antennas", Bell Labs. Technical Journal, vol. 1, no.2, 1996, pp41-59.

[86] D. Gesbert and M. Shafi and D. S. Shiu and P. J. Smith and A. Naguib, "From Theory to Practice: An Overview of MIMO Space-Time Coded Wireless Systems", IEEE J. Sel. Areas Commun., vol. 21, no.3, 2003, pp281-302.

[87] R. A. Soni and R. M. Buehrer, "On the Performance of Open-Loop Transmit Diversity Techniques for IS-2000 Systems: A Comparative Study", IEEE Trans. Wireless Commun., vol. 3, no.5, 2004, pp16021615.

[88] V. Tarokh and A. Naguib and N. Seshadri and A.R. Calderbank, "Space-Time Codes for High Data Rate Wireless Communication: Performance Criteria in the Presence of Channel Estimation Errors, Mobility, and Multile Paths", IEEE Trans. Commun., vol. 47, no.2, 1999, pp199-207.

[89] S.X. Ng and L. Hanzo, "On the MIMO Channel Capacity of Multidimensional Signal Sets", IEEE Trans. Veh. Technol., vol. 55, no.2, 2006, pp528-536.

[90] A. Sendonaris and E. Erkip and B. Aazhang, "User Cooperation Diversity-Part I: System Description", IEEE Trans. Commun., vol. 51, no.11, 2003, pp1927-1938.

[91] J. N. Laneman and D. N. C. Tse and G. W. Wornell, "Cooperative Diversity in Wireless Networks: Efficient Protocols and Outage Behavior", IEEE Trans. Inf. Theory, vol. 50, no.12, 2004, pp30623080.

[92] R. Pabst and B. H. Walke and D. C. Schultz and P. Herhold and H. Yanikomeroglu and S. Mukherjee and H. Viswanathan and M. Lott and W. Zirwas and M. Dohler and H. Aghvami and D. D. Falconer and G. P. Fettweis, ”Relay-Based Deployment Concepts for Wireless and Mobile Broadband Radio", IEEE Commun. Mag., vol. 42, no.9, 2004, pp80-89.

[93] H-F Lu and P.V. Kumar, "Rate-Diversity Tradeoff of Space-Time Codes With Fixed Alphabet and Optimal Constructions for PSK Modulation”, IEEE Trans. Inf. Theory, vol. 49, no.10, 2003, pp27472751.

[94] J. Moon and Y. Lee, "Rapid Slot Synchronization in the Presence of Large Frequency Offset and Doppler Spread in W-CDMA Systems", IEEE J. Wireless Commun., vol. 4, no.4, 2005, pp1325-1330.

[95] S. Fukumoto, K. Higuchi, M. Sawahashi and F. Adachi, ”Experiments on Space Time Block Coding Transmit Diversity (STTD) in WCDMA Forward Link", IEICE Transactions on Fundamentals, vol. E84-A, no.12, 2001, pp3045-3057.

[96] 3GPP, "3GPP TS 25.211 V7.0.0, Physical Channels and Mapping of Transport Channels onto Physical Channels (FDD)", 3GPP, 2006.

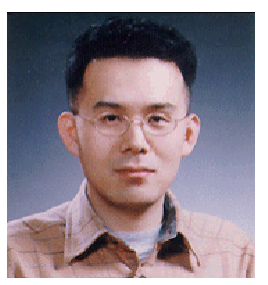

SeungHwan Won (M'04) received the B.S. and M.S. degrees in Radio Science and Engineering from Korea University, Seoul, Republic of Korea, in 1999 and 2001, respectively. He was a research engineer in Mobile Communication Technology Research Lab, LG Electronics R\&D, from January 2001 to September 2004. He was the recipient of the 2004 state scholarship of the Information and Telecommunication National Scholarship Program, Ministry of Information and Communication (MIC), Republic of Korea. During 2004 and 2008 he conducted research, working towards the Ph.D. degree in the Communications Research Group, School of Electronics and Computer Science at the University of Southampton, UK. His major research interests included initial synchronization in non-coherent MIMO aided single- and multi-carrier CDMA, IDMA and OFDMA as well as in iterative synchronization schemes designed for MIMO aided single- and multi-carrier transmission systems. He published a host of papers in these research fields. Upon completing his $\mathrm{PhD}$ he returned to his native Korea and joined Samsung.

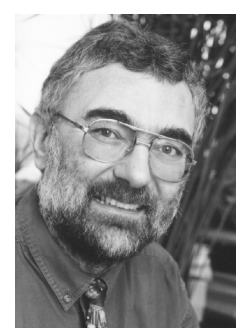

Lajos Hanzo, Fellow of the Royal Academy of Engineering, received his first-class degree in electronics in 1976 and his doctorate in 1983. In 2004 he was awarded the Doctor of Sciences (DSc) degree by the Uniersity of Southampton, UK. During his career in telecommunications he has held various research and academic posts in Hungary, Germany and the UK. Since 1986 he has been with the Department of Electronics and Computer Science, University of Southampton, UK, where he holds the chair in telecommunications. He co-authored 20 John Wiley and IEEE Press books totalling in excess of 10000 pages on mobile radio communications and published about 950 research papers and book chapters at IEEE Xplore. He is also active in organizing and chairing IEEE conferences, such as WCNC 2006, WCNC 2009, VTC 2010 Spring, VTC 2010 Fall, VTC 2011 Spring, etc. He also presented keynote and overview lectures and has been awarded a number of distinctions. Currently he heads an academic research team, working on a range of research projects in the field of wireless multimedia communications sponsored by industry, the Engineering and Physical Sciences Research Council (EPSRC) UK, the European FP 7 Programme and the Mobile Virtual Centre of Excellence (VCE), UK. He is an enthusiastic supporter of industrial and academic liaison and he offers a range of industrial courses. Lajos is also an IEEE Distinguished Lecturer and a Governor of both the Communications as well as the Vehicular Technology Society, a Fellow of both the IEEE and the IET/IEE. He is an editorial board member of the Proceedings of the IEEE. He is the Editor-in-Chief of the IEEE Press. For further information on research in progress and associated publications please refer to http://www-mobile.ecs.soton.ac.uk 